\title{
Bioindication of Ecological State and Water Quality by Phytoplankton in the Shardara Reservoir, Kazakhstan
}

\author{
Sophia Barinova ${ }^{1, *}$, Elena Krupa ${ }^{2}$ \\ ${ }^{1}$ Institute of Evolution, University of Haifa, Mount Carmel, Israel \\ ${ }^{2}$ Republican State Enterprise on the Right of Economic Use "Institute of Zoology", Ministry of Education and Science, Science Committee, \\ Almaty, Republic of Kazakhstan
}

Copyright $\mathrm{C} 2017$ by authors, all rights reserved. Authors agree that this article remains permanently open access under the terms of the Creative Commons Attribution License 4.0 International License

\begin{abstract}
Altogether 76 species of algae were revealed in 13 sampling stations of the Shardara Reservoir's phytoplankton during summer 2015. Chlorophyta algae prevailed. Bioindication methods were used to characterize the reservoir water quality and trophic level as fresh and mesotrophic. For the first time, we used a new statistical approach in analyzing data on biodiversity and phytoplankton productivity in order to determine the main source of pollution. The spatial maps for different variables together with bioindication results were drawn using the Statistica 12.0 program. As an introduction to the new surface mapping method, we conclude that statistical maps are more representative and correspond better with the environment. The maps helped us to characterize the Shardara Reservoir as freshwater, with the source of pollution coming from the rivers and agricultural channels together with some toxic substances being dissolved at the lower part of the waterbody near the dam. The main self-purification processes in the studied waterbody mostly occur by means of the green planktonic algae at the central part of the reservoir. The present study demonstrated that using the surface mapping method is advisable in assessing the ecological conditions of the waterbody not for individual stations but for the reservoir as a whole. This study confirms our bioindication results on the correlation of water temperature and total dissolved solids, which is strongly related to water mass disturbance by the wind. Statistical mapping also confirms that the bioindication method showed a more detailed picture than standard physico-chemical analysis.
\end{abstract}

Keywords Phytoplankton, Bioindication, Statistics, Water Quality, Shardara Reservoir, Kazakhstan

\section{Introduction}

The water source in arid and semi-arid regions of the world is very important. Its volume and quality are monitored by a state's national system and used for settlements and agricultural purposes. How to preserve this water source in good condition is vital. Therefore, responsible governments try to find the sources of water pollution.

The Shardara Reservoir in the arid zone of southern Kazakhstan was formed in 1965 on the Syr-Darya River canal by means of a dam. Its length is up to $80 \mathrm{~km}$ at maximum filling, with a maximum width of $25 \mathrm{~km}$. The reservoir is elongated from north-west to south-west. The right bank is leveled, composed of loose sandy clay and clay loam, with steep underwater ledges. The left Bank is flat, dissected with bays and coves.

Research on the hydrochemical mode and toxic pollution of the reservoir began since its formation [1-4]. High concentrations of heavy metals and pesticides in the water were reported until the mid-90s. In the subsequent period of economic recession, the amount of utilized pesticides was reduced, which led to the improvement of the ecological situation in the region.

The Shardara Reservoir is one of the largest fisheries in Kazakhstan, therefore, monitoring its ecological status and identification of its main sources of pollution are deemed urgent. Actually, the biota-environment relationship reveals some important properties of studied ecosystem which reflect major factors influenced the reservoir water quality. Different methods of this relationships analysis can be used, but we prefer the integral approaches that can give total picture of the reservoir ecosystem state. The integral bioindication methods on the base of algal ecology have large experience [5] with a final result as series of the river basin water quality maps.

In the same time, the integral methods for the reservoir water quality mapping are not so developed. The map-making process usually attempts to make visible the association between different variables of landscape and biota [6]. The results are usable in optimizing of agriculture process. Second usual approach is represented results of hand-drawn mapping from the data of observation, and 
represents not exact measurements, but has a purpose to represent features in the landscape [7]. Up to now used the advanced cartographic method on the base of remotely sensed data which have a GIS coordinates [8]. This type of constructions can be used for large-scale community mapping in purpose to reveal some differences in the analyzed landscape. In our opinion, these above mentioned mapping methods cannot give enough information for the reservoir water quality and pollution source analysis. In any case, mentioned types of mapping require special data mining and special programs as well as reflect the collected data only without prognostic properties.

Thus, the aim of this work is to reveal the algal species in the phytoplankton communities of the Shardara reservoir, which are used to characterize its water quality by bioindication methods, as well as to reveal the major sources of water pollution in the reservoir's surface by means of new statistical methods of spatial analysis.

\section{Materials and Methods}

\subsection{Sampling and Chemical analysis}

Research on salinity and chemical composition was carried out in June 2015 in 3 stations, and research of the content of biogenic elements, heavy metals and organochlorine pesticides in the water was carried out by means of a grid of 13 stations (Fig. 1). The measurements of temperature and $\mathrm{pH}$ values of the surface water layers were taken in the field by using the Hanna HI 98129 instrument. Water clarity was measured with a Secchi disk. Coordinate referencing of the stations was done by Garmin eTrex GPS-navigator. Conventional methods of chemical analysis of water were used [9-10]. Water samples were analyzed by 3-4 replications.

The settling method was used [11] to process phytoplankton samples. Species identification of planktonic algae was performed using determinant handbooks for relevant divisions [12-17].

\subsection{Bioindication}

The ecological characteristics of algal species were obtained from the database compiled for freshwater algae of the world from multiple analyses of algal biodiversity by S.S. Barinova et al. [18], according to substrate preference, temperature, oxygenation, $\mathrm{pH}$, salinity, organic enrichments, N-uptake metabolism, and trophic states. The ecological groups were separately assessed according to their significance for bioindications. Species that respond predictably to environmental conditions were used as bioindicators for particular variables of aquatic ecosystems, the dynamics of which are related to environmental changes.

\subsection{Statistical methods}

The statistical methods used were those recommended by Heywood [19] for the development of taxonomic studies, namely, the GRAPHS program [20] for comparative floristics. The innovative approach with Surface plots' construction was applied using the Statistica 12.0 program for analysis of biological and environmental variables' relationship.

\subsection{Description of the Study Site}

Potential sources of pollution of the reservoir are: the transit runoff of the trans-boundary Syr-Darya River from Uzbekistan territory, collector and drainage water and non-point surface runoff as well as effluent waters. There are tracts of irrigated land in 9.4 thousand hectares along the Keles River, a tributary to the Syr-Darya River upstream of the reservoir (Fig. 1a). Approximately 136 thousand hectares of agricultural land (mainly cotton and corn) are located on the left bank of the upper and middle part of the reservoir (Fig. 1b), which is irrigated through the Dostyk canal that originates in the Uzbekistan territory. Irrigational water withdrawal also occurs directly from the reservoir using a mechanical delivery into the Dostyk canal (Fig. 1e). Drainage water return is discharged into Arnasay Bay (Fig. 1d). On the right bank, there are small tracts of melons fields (Fig. 1c) that are watered through pipes drawing water from the reservoir. Small and large domestic animals - sheep, goats, cows, and horses graze here.

\section{Results}

\subsection{Chemical Composition of the Reservoir Water}

Total Dissolved Solids (TDS) and chemical composition of the water in the Shardara Reservoir in 2015 is represented in Table 1.

Total ionic content of water varied over the water area to a small extent, slightly increasing the influence area of Syr-Darya River runoff and reaching an average of 1055 $\mathrm{mg} \mathrm{dm}{ }^{-3}$. The total content of dissolved solids revealed the water was brackish [21], with the predominance of sulfate and sodium ions. In the influence area of river runoff, the amount of sodium, magnesium, calcium, and sulfates slightly increased. The highest concentrations of potassium were registered along the left bank of the reservoir.

The concentration of biogenic compounds in the water was at a low level (Table 2) and did not exceed the maximum permissible concentration prescribed for fishery water bodies [22].

The content of phosphate in water varied within a small range. Therefore, the reservoir water can be characterized as low-alkaline, with a concentration of sodium sulfates, and brackish.

\subsection{Phytoplankton}

Phytoplankton of the Shardara Reservoir in summer 2015 was represented by 76 species (Table 3). The largest diversity showed green algae (Fig. 2a) whereas the diatoms held second place.

The number of species in the phytoplankton community 
ranged from 20 to 37 across the aquatic area. The highest total diversity of planktonic algae was found in the upper part of the reservoir (Fig. 2b) except for dinophytes, which showed higher diversity in the deep-water zone near the dam (Fig. 3).

Diatoms were the most diverse along the right bank of the reservoir; the blue-green algae as well as green algae, on the other hand, were located along the left. Maximum diversity of Dinophyta and Charophyta algae was formed in controversial parts of the reservoir (Fig. 3).
Analysis of species composition of phytoplankton communities by methods of comparative floristic similarity showed two clusters could be distinguished at the 50\% similarity: 1) more similar to each other at stations 10, 12, and 13 in the shallow upper part of the reservoir, and 2) other stations (Fig. 4). The communities were richer in the first cluster, comprising 29-37 species, in the second cluster - less rich, with 20-30 species. However, in both groups, green algae dominated in terms of diversity.

Table 1. Total Dissolved Solids (TDS) and chemical composition $\left(\mathrm{mg} \mathrm{l}^{-3}\right)$ of Shardara Reservoir, summer 2015.

\begin{tabular}{|c|c|c|c|}
\hline Indicator, Station & 1 & 5 & 12 \\
\hline $\mathrm{Ca}$ & 109.2 & 104.2 & 110.2 \\
\hline $\mathrm{Mg}$ & 59.6 & 59.6 & 115.7 \\
\hline $\mathrm{Na}+\mathrm{K}$ & 120.2 & 173.9 & 177 \\
\hline $\mathrm{HCO}_{3}$ & 180 & 477.5 & 515.3 \\
\hline $\mathrm{SO}_{4}$ & 489.8 & 78 & 78 \\
\hline $\mathrm{Cl}$ & 79.8 & 1025 & 1083 \\
\hline $\mathrm{TDS}$ & 1056 & 110 & 120 \\
\hline $\mathrm{Na}$ & 115 & 5.7 & 5 \\
\hline $\mathrm{K}$ & 5.2 & & 5 \\
\hline
\end{tabular}

Table 2. Averaged chemical data $\left(\mathrm{mg} \mathrm{l}^{-3}\right), \mathrm{pH}$, and transparency by Secchi disk (m) over the sampling stations in the Shardara Reservoir in summer 2015 with GIS coordinates

\begin{tabular}{|c|c|c|c|c|c|c|c|c|}
\hline St. & North & East & $\mathrm{NH}_{4}$ & $\mathrm{NO}_{3}$ & $\mathrm{NO}_{2}$ & $\mathrm{P}_{4} \mathrm{PO}_{4}$ & $\mathrm{pH}$ & $\mathrm{Secchi}(\mathrm{S})$ \\
\hline 1 & 41.25 & 67.97 & 0.100 & 5.540 & 0.060 & 0.020 & 8.6 & 2.3 \\
\hline 2 & 41.24 & 67.96 & 0.096 & 5.020 & 0.044 & 0.017 & 8.6 & 2.7 \\
\hline 3 & 41.24 & 67.92 & 0.100 & 5.815 & 0.062 & 0.025 & 8.5 & 3.2 \\
\hline 4 & 41.21 & 67.93 & 0.040 & 5.760 & 0.050 & 0.020 & 8.5 & 2.0 \\
\hline 5 & 41.10 & 68.00 & 0.050 & 4.650 & 0.035 & 0.020 & 8.6 & 1.7 \\
\hline 6 & 41.14 & 68.04 & 0.200 & 6.200 & 0.060 & 0.020 & 8.5 & 1.3 \\
\hline 7 & 41.17 & 68.07 & 0.196 & 5.815 & 0.023 & 0.007 & 8.5 & 1.5 \\
\hline 8 & 41.17 & 68.10 & 0.300 & 5.540 & 0.060 & 0.020 & 8.5 & 1.3 \\
\hline 9 & 41.15 & 68.21 & 0.085 & 5.020 & 0.370 & 0.170 & 8.6 & 1.0 \\
\hline 10 & 41.08 & 68.22 & 0.085 & 5.020 & 0.174 & 0.078 & 8.6 & 0.5 \\
\hline 11 & 41.10 & 68.26 & 0.050 & 4.870 & 0.085 & 0.030 & 8.6 & 0.7 \\
\hline 12 & 41.12 & 68.29 & 0.100 & 5.760 & 0.085 & 0.050 & 8.5 & 0.5 \\
\hline 13 & 41.12 & 68.35 & 0.096 & 5.815 & 0.091 & 0.039 & 8.5 & 0.4 \\
\hline
\end{tabular}


Table 3. Species composition and ecological characteristics of phytoplankton of the Shardara Reservoir, summer 2015

\begin{tabular}{|c|c|c|c|c|c|c|c|c|c|c|c|}
\hline Taxa & $\mathrm{T}$ & $\mathrm{Hab}$ & Reo & $\mathrm{pH}$ & pH-range & Hal & $\mathrm{D}$ & Tro & Aut-Het & $\mathrm{s}$ & Sap \\
\hline \multicolumn{12}{|l|}{ Cyanobacteria } \\
\hline Aphanocapsa delicatissima West \& G.S.West & - & P-B & - & - & - & $\mathrm{i}$ & - & $\mathrm{m}$ & - & - & - \\
\hline $\begin{array}{l}\text { Aphanocapsa incerta (Lemmermann) G. } \\
\text { Cronberg \& Komárek }\end{array}$ & - & P-B & - & - & - & $\mathrm{i}$ & - & me & - & 2.2 & $\mathrm{~b}$ \\
\hline Merismopedia minima G. Beck & - & B.S & aer & - & - & - & - & o & - & - & - \\
\hline Merismopedia punctata Meyen & - & P-B & - & ind & - & $\mathrm{i}$ & - & me & - & 1.8 & $\mathrm{o}-\mathrm{a}$ \\
\hline Microcystis aeruginosa (Kützing) Kützing & - & $\mathrm{P}$ & - & - & - & hl & - & $\mathrm{e}$ & - & 2.1 & $\mathrm{~b}$ \\
\hline Oscillatoria planctonica Woloszynska & - & $\mathrm{P}$ & - & - & - & $\mathrm{i}$ & - & me & - & 1.5 & o-b \\
\hline $\begin{array}{l}\text { Planktolyngbya limnetica (Lemmermann) } \\
\text { Komárková-Legnerová \& Cronberg }\end{array}$ & - & P-B.S & st-str & - & - & $\mathrm{hl}$ & - & $\mathrm{e}$ & - & 1.5 & o-b \\
\hline Trichodesmium lacustre Klebahn & - & $\mathrm{P}$ & st & - & - & - & - & - & - & - & - \\
\hline \multicolumn{12}{|l|}{ Bacillariophyta } \\
\hline Achnanthes gibberula Grunow & eterm & $\mathrm{B}$ & st-str & ind & - & $\mathrm{mh}$ & sx & $\mathrm{o}-\mathrm{m}$ & - & 0.3 & $\mathrm{x}$ \\
\hline Amphora ovalis (Kützing) Kützing & temp & $\mathrm{B}$ & st-str & alf & $6.2-9.0$ & $\mathrm{i}$ & sx & me & ate & 1.5 & o-b \\
\hline Cocconeis placentula Ehrenberg & temp & P-B & st-str & alf & $5.5-9.0$ & $\mathrm{i}$ & es & me & ate & 1.35 & o \\
\hline $\begin{array}{c}\text { Ctenophora pulchella (Ralfs ex Kützing) } \\
\text { D.M.Williams \& Round }\end{array}$ & - & P-B & st-str & alf & - & $\mathrm{i}$ & - & $\mathrm{o}-\mathrm{m}$ & ate & 2.3 & $\mathrm{~b}$ \\
\hline Cyclotella meneghiniana Kützing & temp & P-B & st & alf & $5.5-9.0$ & hl & $\mathrm{sp}$ & $\mathrm{e}$ & hne & 2.8 & $\mathrm{a}-\mathrm{o}$ \\
\hline Fragilaria acus (Kützing) Lange-Bertalot & - & $\mathrm{P}$ & st-str & alb & - & $\mathrm{i}$ & es & - & - & 1.8 & $\mathrm{o}-\mathrm{a}$ \\
\hline Fragilaria capucina Desmazières & - & P-B & - & ind & 7.7 & $\mathrm{i}$ & es & $\mathrm{m}$ & - & 1.6 & b-o \\
\hline Fragilaria construens (Ehrenberg) Grunow & temp & P-B & st-str & alf & $5.5-9.0$ & $\mathrm{i}$ & sx & me & ats & 1.3 & o \\
\hline Fragilaria crotonensis Kitton & - & $\mathrm{P}$ & st-str & alf & 7.5 & I & es & $\mathrm{m}$ & ate & 1.5 & $\mathrm{o}-\mathrm{b}$ \\
\hline Gomphonema parvulum (Kützing) Kützing & temp & $\mathrm{B}$ & str & ind & 4.5 & $\mathrm{i}$ & es & $\mathrm{o}-\mathrm{m}$ & hne & 2.35 & $\mathrm{~b}$ \\
\hline $\begin{array}{l}\text { Lindavia comta (Kützing) Nakov, Gullory, } \\
\text { Julius, Theriot \& Alverson }\end{array}$ & - & $\mathrm{P}$ & st & alf & 7.2 & i & sx & $\mathrm{o}-\mathrm{m}$ & - & 1.2 & o \\
\hline Melosira varians C.Agardh & temp & P-B & st-str & ind & $5.0-9.0$ & hl & es & me & hne & 2.1 & $\mathrm{~b}$ \\
\hline Nitzschia acicularis (Kützing) W.Smith & temp & P-B & - & alf & $7.85-8.15$ & $\mathrm{i}$ & es & $\mathrm{e}$ & hce & 2.7 & $\mathrm{a}-\mathrm{o}$ \\
\hline Proschkinia longirostris (Hustedt) D.G.Mann & - & $\mathrm{B}$ & - & alf & - & $\mathrm{hl}$ & - & - & - & - & - \\
\hline Thalassiosira lacustris (Grunow) Hasle & - & $\mathrm{P}$ & - & - & - & hl & $\mathrm{sp}$ & - & - & 1.4 & $\mathrm{o}-\mathrm{b}$ \\
\hline Ulnaria ulna (Nitzsch) P. Compère & temp & P-B & st-str & ind & $5.0-9.2$ & $\mathrm{i}$ & es & o-e & ate & 2.25 & $\mathrm{~b}$ \\
\hline \multicolumn{12}{|l|}{ Ochrophyta (Chrysophyta) } \\
\hline Dinobryon elegans Reverdin & - & - & - & - & - & - & - & - & - & - & - \\
\hline Dinobryon sertularia Ehrenberg & - & $\mathrm{P}$ & - & - & - & $\mathrm{i}$ & - & - & - & 1.3 & o \\
\hline Mallomonas sp. & - & - & - & - & - & - & - & - & - & - & - \\
\hline \multicolumn{12}{|l|}{ Miozoa (Dinophyta) } \\
\hline Ceratium hirundinella (O.F.Müller) Dujardin & - & $\mathrm{P}$ & st-str & - & - & $\mathrm{i}$ & - & - & - & 1.3 & o \\
\hline Gonyaulax apiculate Entz & - & - & - & - & - & - & - & - & - & 1.1 & o \\
\hline Gymnodinium variabile Herdman & - & - & - & - & - & - & - & - & - & - & - \\
\hline $\begin{array}{c}\text { Peridiniopsis penardii (Lemmermann) } \\
\text { Bourrelly }\end{array}$ & - & $\mathrm{P}$ & - & - & - & hl & - & - & - & 1.3 & o \\
\hline $\begin{array}{c}\text { Peridiniopsis polonica (Woloszynska) } \\
\text { Bourrelly }\end{array}$ & - & $\mathrm{P}$ & st & - & - & - & - & - & - & 1.3 & o \\
\hline Peridiniopsis quadridens (Stein) Bourrelly & - & - & - & - & - & - & - & - & - & - & - \\
\hline Prorocentrum lima (Ehrenberg) F.Stein & - & - & - & - & - & - & - & - & - & - & - \\
\hline \multicolumn{12}{|l|}{ Chlorophyta } \\
\hline $\begin{array}{c}\text { Acutodesmus acuminatus (Lagerheim) } \\
\text { P.M.Tsarenko }\end{array}$ & - & P-B & st-str & ind & - & $\mathrm{i}$ & - & - & - & 2.15 & $\mathrm{~b}$ \\
\hline Chlorella vulgaris Beyerinck & - & $\begin{array}{l}\text { P-B. } \\
\text { pb.S }\end{array}$ & - & - & - & $\mathrm{hl}$ & - & - & - & 3.1 & a \\
\hline Chlorococcum infusionum (Schrank) Meneghini & - & P.S & st & - & - & - & - & - & - & 2.7 & a-o \\
\hline Chlorolobion braunii (Nägeli) Komárek & - & P-B & st & - & - & - & - & - & - & 1.5 & o-b \\
\hline Chodatellopsis elliptica Korshikov & - & - & - & - & - & - & - & - & - & - & - \\
\hline Coelastrumm icroporum Nägeli & - & P-B & st-str & ind & - & $\mathrm{i}$ & - & - & - & 2.3 & $\mathrm{~b}$ \\
\hline Coelastrum sphaericum Nägeli & - & P-B & st-str & - & - & $\mathrm{i}$ & - & - & - & 1.4 & o-b \\
\hline
\end{tabular}




\begin{tabular}{|c|c|c|c|c|c|c|c|c|c|c|c|}
\hline Coenochloris mucosa (Korshikov) Hindák & - & - & - & - & - & - & - & - & - & - & - \\
\hline Coenochloris pyrenoidosa Korshikov & - & $\mathrm{P}$ & - & - & - & hl & - & - & - & - & - \\
\hline Coenocystis planctonica Korshikov & - & $\mathrm{P}$ & - & - & - & $\mathrm{i}$ & - & - & - & 2 & $\mathrm{~b}$ \\
\hline $\begin{array}{l}\text { Coenolamellus botryoideus A. I. } \\
\text { Proshkina-Lavrenko }\end{array}$ & - & - & - & - & - & - & - & - & - & - & - \\
\hline $\begin{array}{c}\text { Crucigenia emarginata } \text { (West \& G. S. West) } \\
\text { Schmidle }\end{array}$ & - & $\mathrm{P}$ & st & - & - & - & - & - & - & - & - \\
\hline Crucigenia quadrata Morren & - & P-B & st-str & acf & - & $\mathrm{i}$ & - & - & - & 1.9 & $\mathrm{o}-\mathrm{a}$ \\
\hline $\begin{array}{c}\text { Crucigeniella irregularis (Wille) P. M. } \\
\text { Tsarenko \& D.M.John }\end{array}$ & - & P-B & st-str & ind & - & $\mathrm{i}$ & - & - & - & - & - \\
\hline $\begin{array}{c}\text { Desmodesmus communis (E. Hegewald) E. } \\
\text { Hegewald }\end{array}$ & - & P-B & st-str & ind & - & $\mathrm{i}$ & - & - & - & 2.15 & $\mathrm{~b}$ \\
\hline Kirchneriella lunaris (Kirchner) Möbius & - & P-B & st-str & - & - & $\mathrm{i}$ & - & - & - & 1.8 & o-a \\
\hline Lagerheimia marssonii Lemmermann & - & - & - & - & - & - & - & - & - & 2.1 & $\mathrm{~b}$ \\
\hline $\begin{array}{l}\text { Lagerheimia quadriseta (Lemmermann) G. M. } \\
\text { Smith }\end{array}$ & - & $P$ & st-str & - & - & $\mathrm{i}$ & - & - & - & 2.2 & $\mathrm{~b}$ \\
\hline Monoraphidium arcuatum (Korshikov) Hindák & - & P-B & st-str & - & - & $\mathrm{i}$ & - & - & - & 2.1 & $\mathrm{~b}$ \\
\hline $\begin{array}{c}\text { Monoraphidium contortum (Thuret) } \\
\text { Komárková-Legnerová }\end{array}$ & - & P-B & st-str & - & - & $\mathrm{i}$ & - & - & - & 2.2 & $\mathrm{~b}$ \\
\hline $\begin{array}{l}\text { Monoraphidium convolutum (Corda) } \\
\text { Komárková-Legnerová }\end{array}$ & - & P-B & st-str & - & - & - & - & - & - & 2.3 & $\mathrm{~b}$ \\
\hline $\begin{array}{l}\text { Monoraphidium griffithii (Berkeley) } \\
\text { Komárková-Legnerová }\end{array}$ & - & P-B & st-str & - & - & $\mathrm{i}$ & - & - & - & 2.2 & $\mathrm{~b}$ \\
\hline $\begin{array}{c}\text { Monoraphidiumminutum (Nägeli) } \\
\text { Komárková-Legnerová }\end{array}$ & - & P-B & st-str & - & - & $\mathrm{i}$ & - & - & - & 2.5 & b-a \\
\hline $\begin{array}{l}\text { Mucidosphaerium pulchellum (H.C.Wood) } \\
\text { C.Bock, Proschold \& Krienitz }\end{array}$ & - & P-B & st-str & ind & - & $\mathrm{i}$ & - & - & - & 2.3 & $\mathrm{~b}$ \\
\hline $\begin{array}{c}\text { Myrmecia irregularis (J.B.Petersen) Ettl \& } \\
\text { Gärtner }\end{array}$ & - & - & - & - & - & - & - & - & - & - & - \\
\hline Oocystis borgei J.W.Snow & - & P-B & st-str & ind & - & $\mathrm{i}$ & - & - & - & 1.9 & $\mathrm{o}-\mathrm{a}$ \\
\hline Oocystis lacustris Chodat & - & P-B & st-str & - & - & hl & - & - & - & 1.7 & b-o \\
\hline Oocystis solitaria Wittrock & - & $\mathrm{P}$ & st & ind & - & $\mathrm{i}$ & - & - & - & 1.7 & $\mathrm{~b}-\mathrm{o}$ \\
\hline Pediastrum boryanum (Turpin) Meneghini & - & P-B & - & - & - & - & - & - & - & - & - \\
\hline Pediastrum duplex Meyen & - & $\mathrm{P}$ & st-str & ind & - & $\mathrm{i}$ & - & - & - & 2.1 & $\mathrm{~b}$ \\
\hline Planctonema lauterbornii Schmidle & - & - & - & - & - & - & - & - & - & 1.8 & $\mathrm{o}-\mathrm{a}$ \\
\hline $\begin{array}{c}\text { Pseudodidymocystis planctonica (Korshikov) E. } \\
\text { Hegewald \& Deason }\end{array}$ & - & - & - & - & - & - & - & - & - & 1.8 & $\mathrm{o}-\mathrm{a}$ \\
\hline $\begin{array}{c}\text { Pseudopediastrum boryanum (Turpin) E. } \\
\text { Hegewald }\end{array}$ & - & P-B & st-str & ind & - & $\mathrm{i}$ & - & - & - & 2.1 & $\mathrm{~b}$ \\
\hline Scenedesmus obliquus (Turpin) Kützing & - & P-B & st-str & ind & - & $\mathrm{i}$ & - & - & - & 2.05 & $\mathrm{~b}$ \\
\hline Schroederia setigera (Schröder) Lemmermann & - & $\mathrm{P}$ & st-str & - & - & $\mathrm{i}$ & - & - & - & 1.7 & b-o \\
\hline Stauridium tetras (Ehrenberg) E. Hegewald & - & P-B & st-str & ind & - & $\mathrm{i}$ & - & - & - & 2.1 & $\mathrm{~b}$ \\
\hline Tetraëdron caudatum (Corda) Hansgirg & - & P-B & st-str & ind & - & $\mathrm{i}$ & - & - & - & 2 & $\mathrm{~b}$ \\
\hline Tetraëdron minimum (A.Braun) Hansgirg & - & P-B & st-str & - & - & $\mathrm{i}$ & - & - & - & 2.1 & $\mathrm{~b}$ \\
\hline $\begin{array}{l}\text { Willea piculate (Lemmermann) D.M.John, M. J. } \\
\text { Wynne \& P. M. Tsarenko }\end{array}$ & - & P-B & st-str & - & - & - & - & - & - & 2.2 & $\mathrm{~b}$ \\
\hline \multicolumn{12}{|l|}{ Charophyta } \\
\hline Cosmarium granatum Brébisson ex Ralfs & - & $\mathrm{B}$ & st-str & ind & - & $\mathrm{i}$ & - & $\mathrm{m}$ & - & 1.2 & o \\
\hline Cosmarium undulatum Corda ex Ralfs & - & P-B & - & acf & - & $\mathrm{i}$ & - & $\mathrm{m}$ & - & - & - \\
\hline \multicolumn{12}{|c|}{$\begin{array}{l}\text { Note: Temperature preferences (T): temp - temperate temperature, eterm - eurythermic, Substrate preferences (Hab): P - planktonic, P-B - } \\
\text { plankto-benthic, B - benthic. Oxygenation and water moving (Reo): st - standing water, str - streaming water, st-str - low streaming water, aer - } \\
\text { aerophiles.Acidity (pH) degree according Hustedt [23]: alf - alkaliphiles, ind - indifferents; acf - acidophiles; alb - alkalibiontes. Halobity degree } \\
\text { according Hustedt [24] (Hal): i - oligohalobes-indifferent, hl - halophiles, mh - mesohalobes. Organic pollution indicators according to Watanabe et } \\
\text { al. [25] (D): sx - saproxenes, es - eurysaprobes, sp - saprophyles. Trophic state (Tro) [26]: o - oligotraphentic; o-m - oligo-mesotraphentic; } \mathrm{m}- \\
\text { mesotraphentic; me - meso-eutraphentic; e - eutraphentic; o-e - oligo-eutraphentic. Nitrogen uptake metabolism (Aut-Het) [26]: ats - } \\
\text { nitrogen-autotrophic taxa, tolerating very small concentrations of organically bound nitrogen; ate - nitrogen-autotrophic taxa, tolerating elevated } \\
\text { concentrations of organically bound nitrogen; hne - facultatively nitrogen-heterotrophic taxa, needing periodically elevated concentrations of } \\
\text { organically bound nitrogen; hce - nitrogen-heterotrophic taxa, needing elevated concentrations of organically bound nitrogen. Species-specific } \\
\text { Index of Saprobity (s). Self-purification zone preferences (Sap): x - xenosaprob; o - oligosaprob; o-b - oligo-beta-msosaprob; o-a - } \\
\text { oligo-alpha-mesosaprob; b - beta-mesosaprob; b-o - beta-oligo-mesosaprob; b-a - beta-alpha-mesosaprob; a-o - alpha-oligo-mesosaprob; a - } \\
\text { alpha-mesosaprob. }\end{array}$} \\
\hline
\end{tabular}




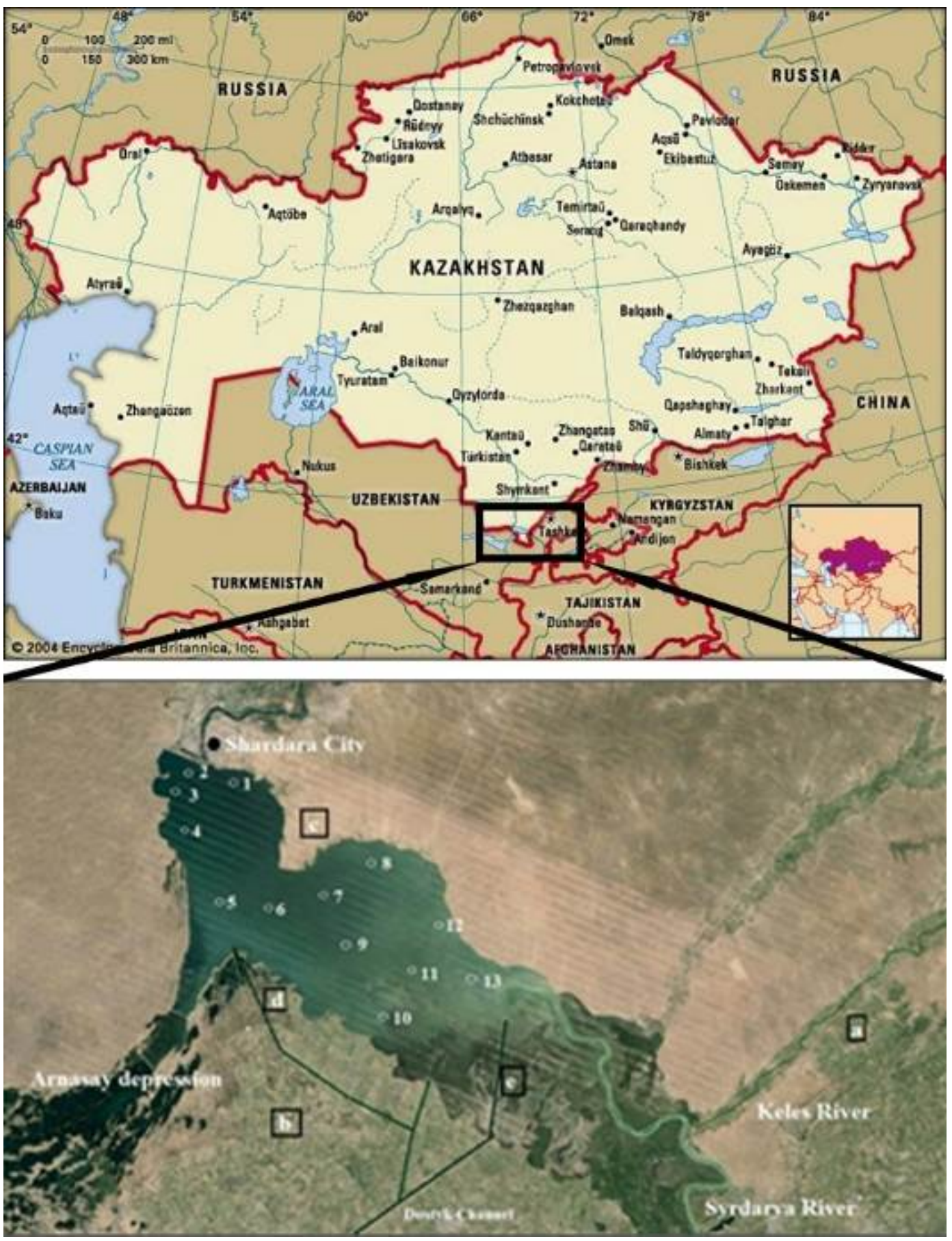

Figure 1. Map of sampling stations in the Shardara Reservoir with potential sources of pollution, summer 2015. Legend: a, b, c - irrigation fields, $d-$ discharge of return drainage water into Gulf Arnasay, e - water taken from Shardara Reservoir; 1-13 - sampling stations 

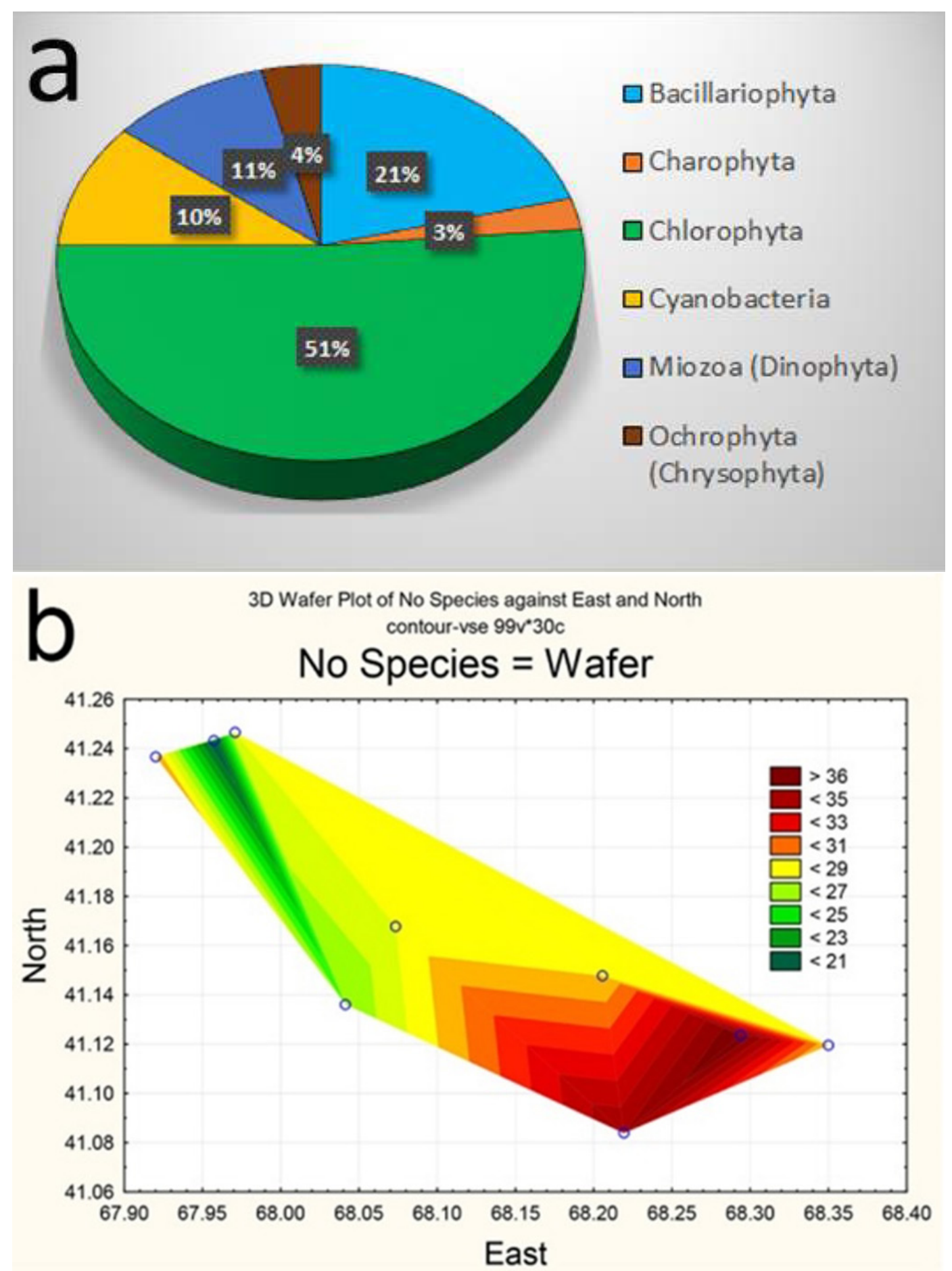

Figure 2. Phytoplankton species diversity over taxonomic divisions (a) and the distribution of phytoplankton species richness (b) across the water surface of the Shardara reservoir, summer 2015. 
a

3D Wafer Plot of Bacillariophyta against East and North
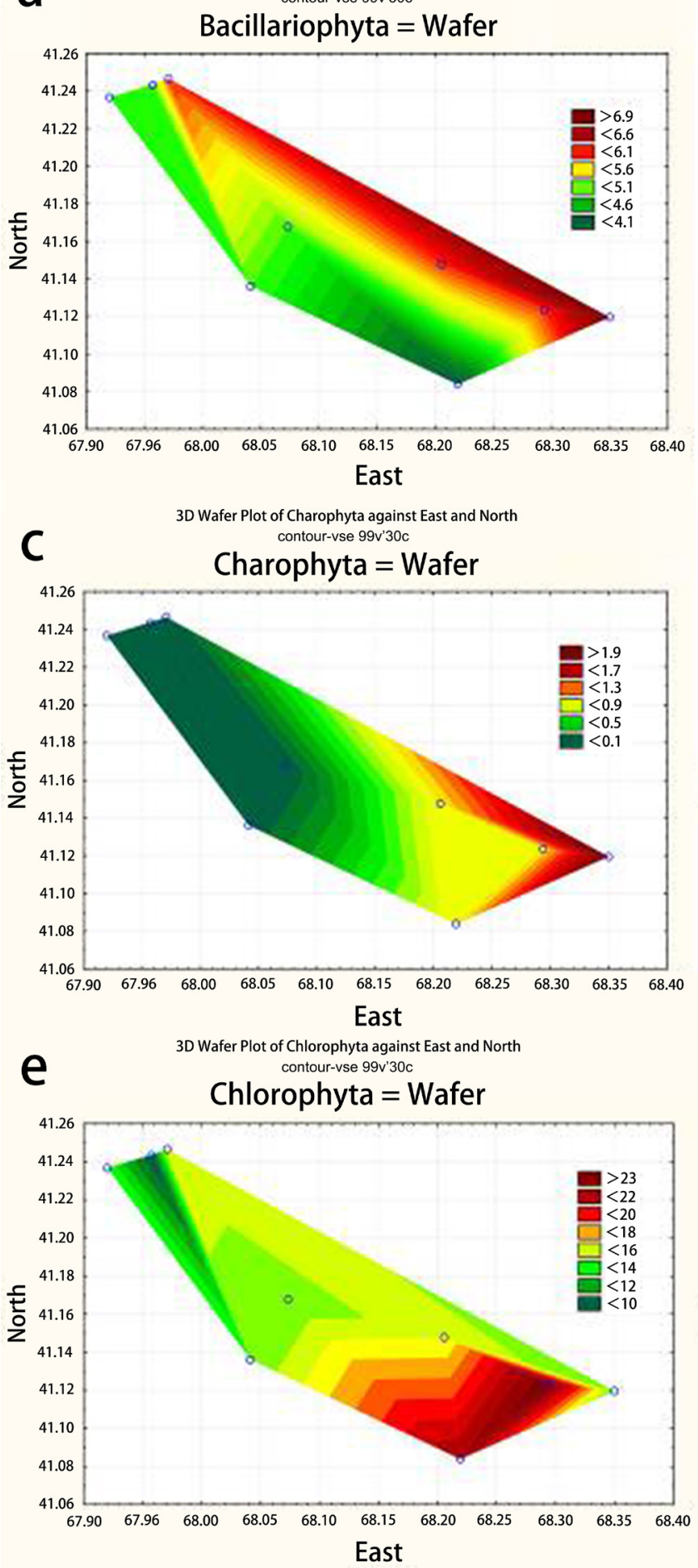
3D Wafer Plot of Cyanobacteria against East and North

Cyanobacteria $=$ Wafer
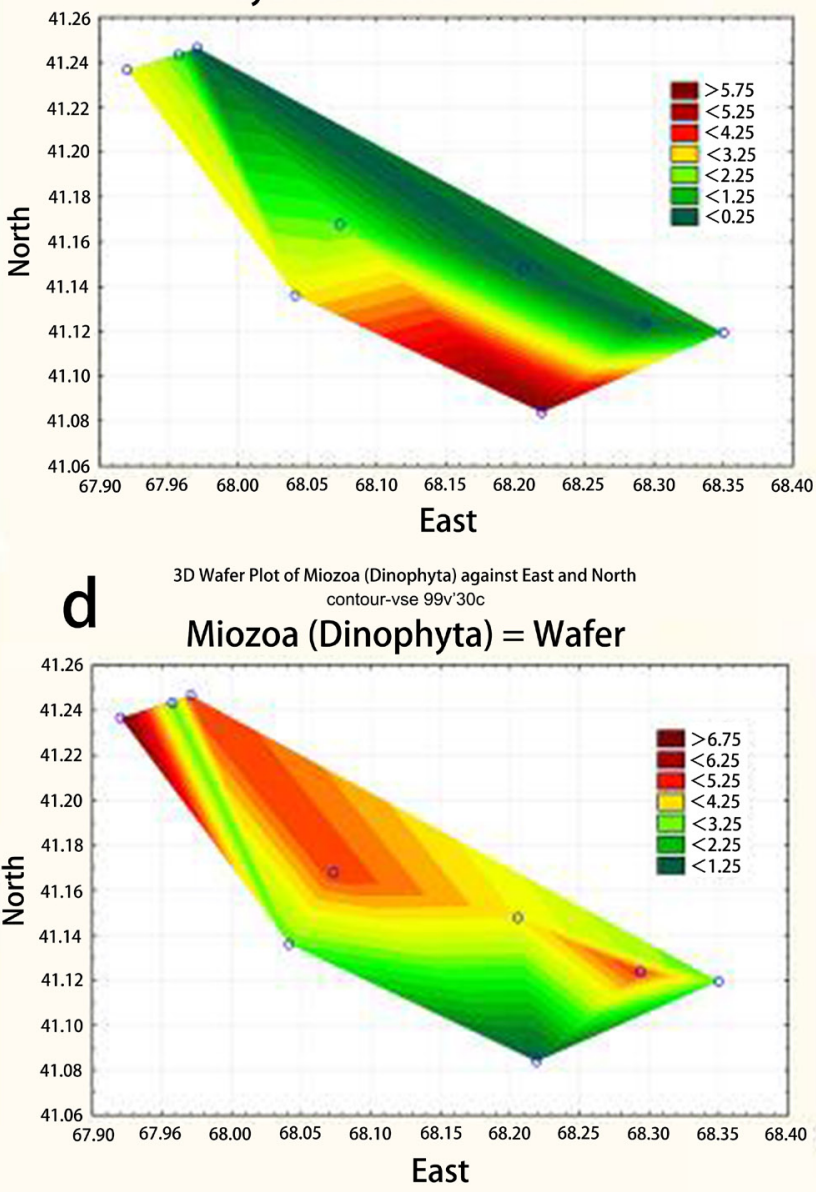

C 3D Wafer Plot of Ochrophyta (Chrysophyta) against East and North Ochrophyta (Chrysophyta) $=$ Wafer

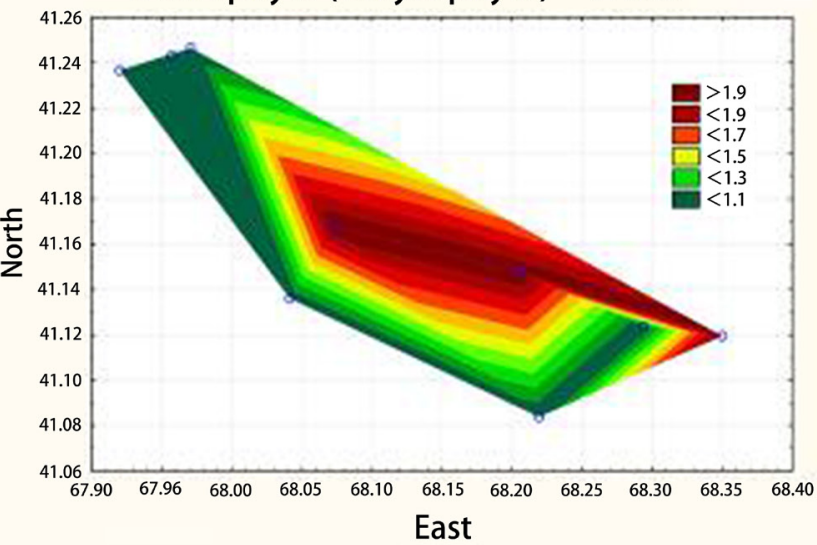

Figure 3. The diversity dynamics of the phytoplankton divisions across the water surface of the Shardara Reservoir, summer 2015. 


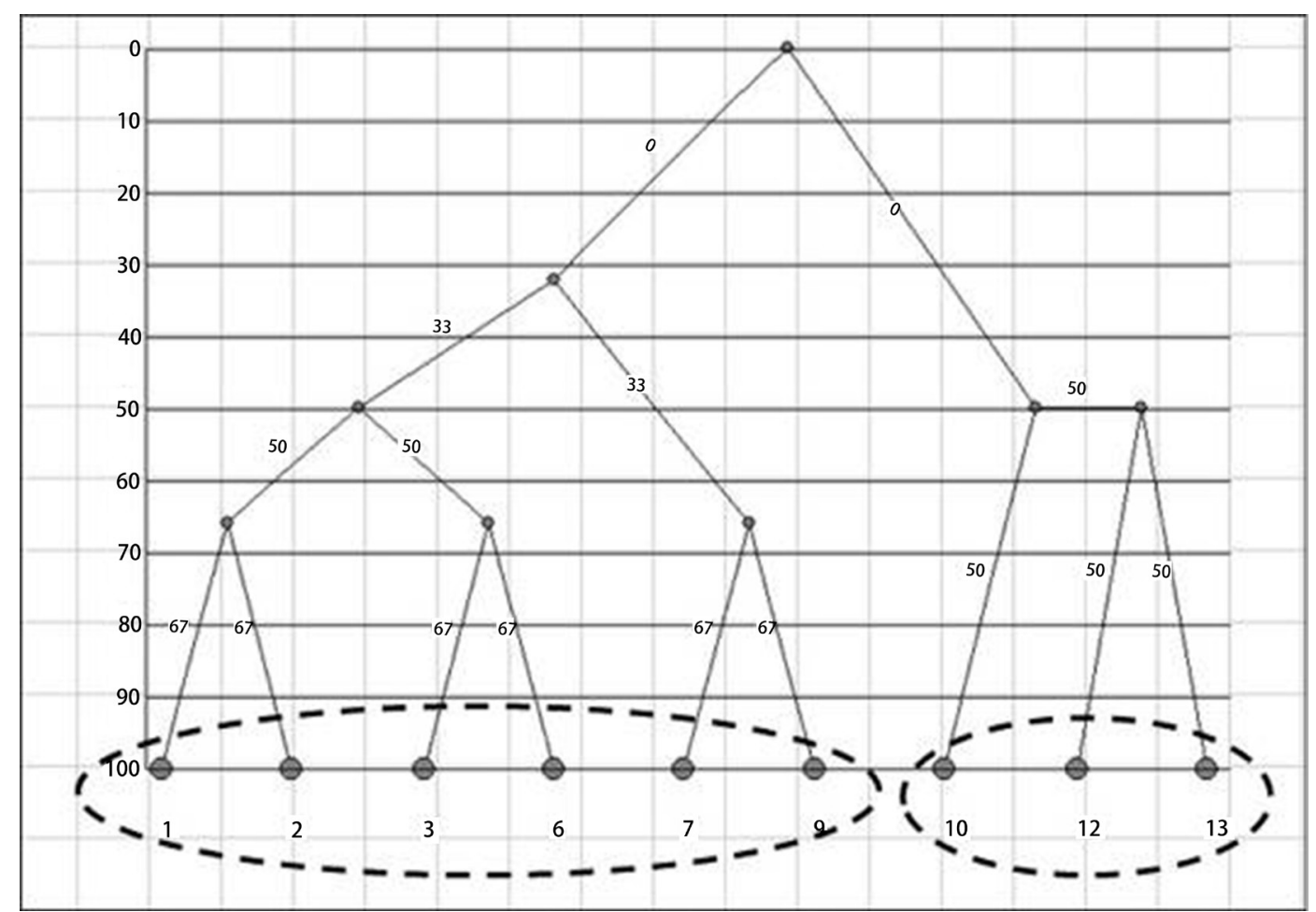

Figure 4. Dendrogram of species richness similarity in phytoplankton communities of the Shardara Reservoir sampling stations during summer 2015

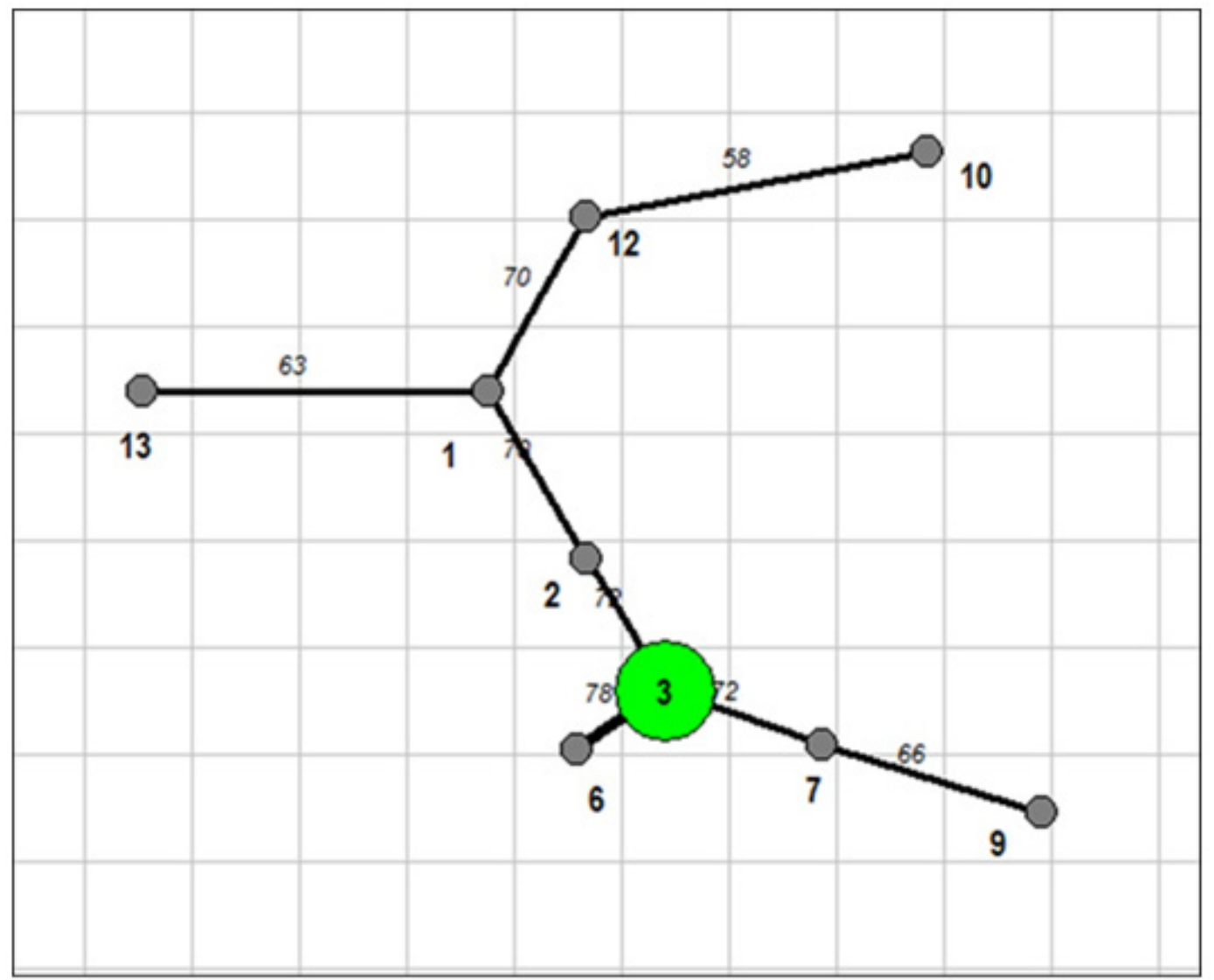

Figure 5. Dendrite of the phytoplankton communities overlapping in the sampling stations of the Shardara Reservoir in summer 2015 


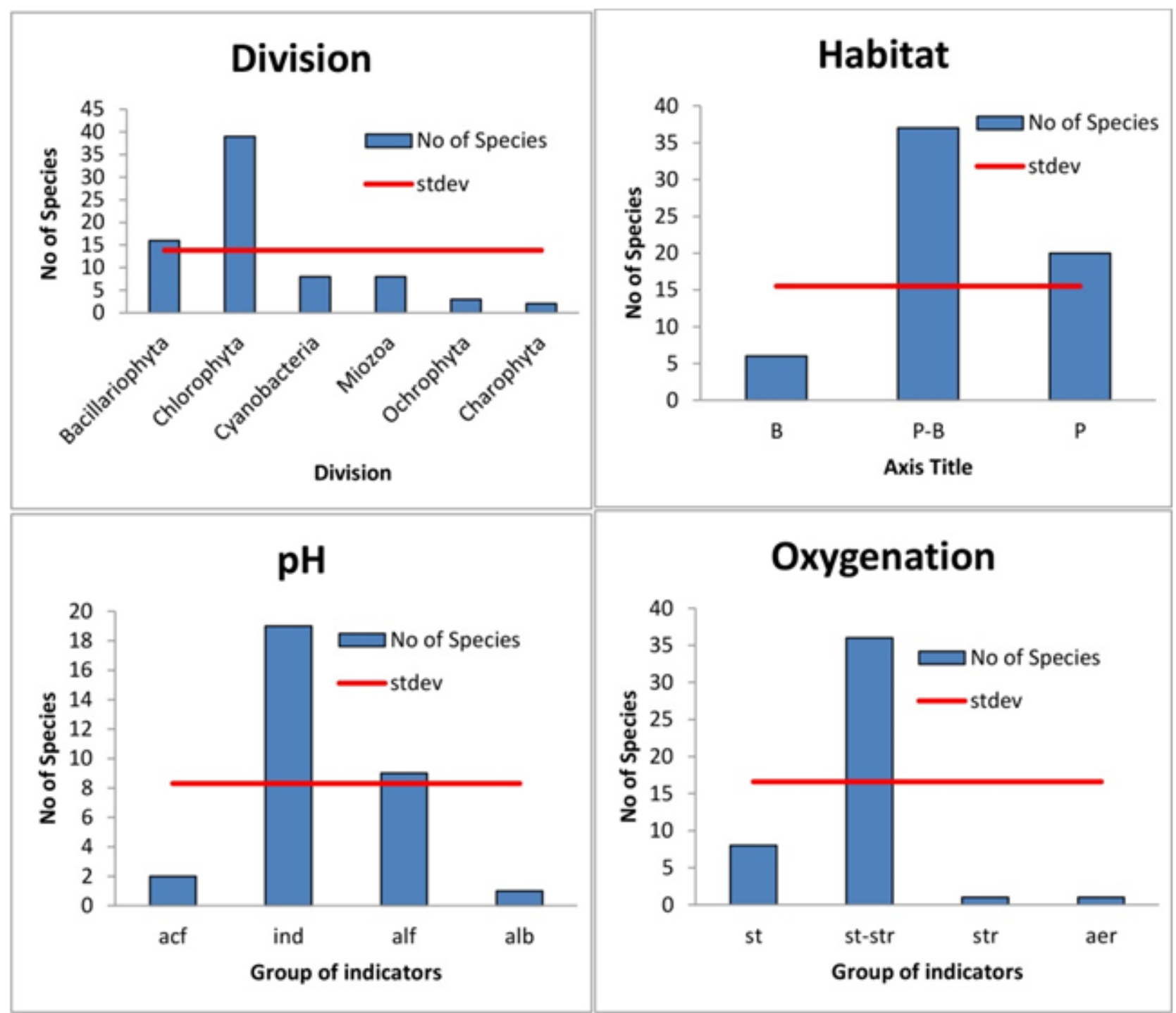

Figure 6. Distribution of the number of species by taxonomic divisions and assessment of the ecological state of the Shardara Reservoir according to phytoplankton species' preferences to habitat type, $\mathrm{pH}$, water mobility and saturation of oxygen, summer 2015 . Abbreviations for indicator groups - appear in Table 3. 


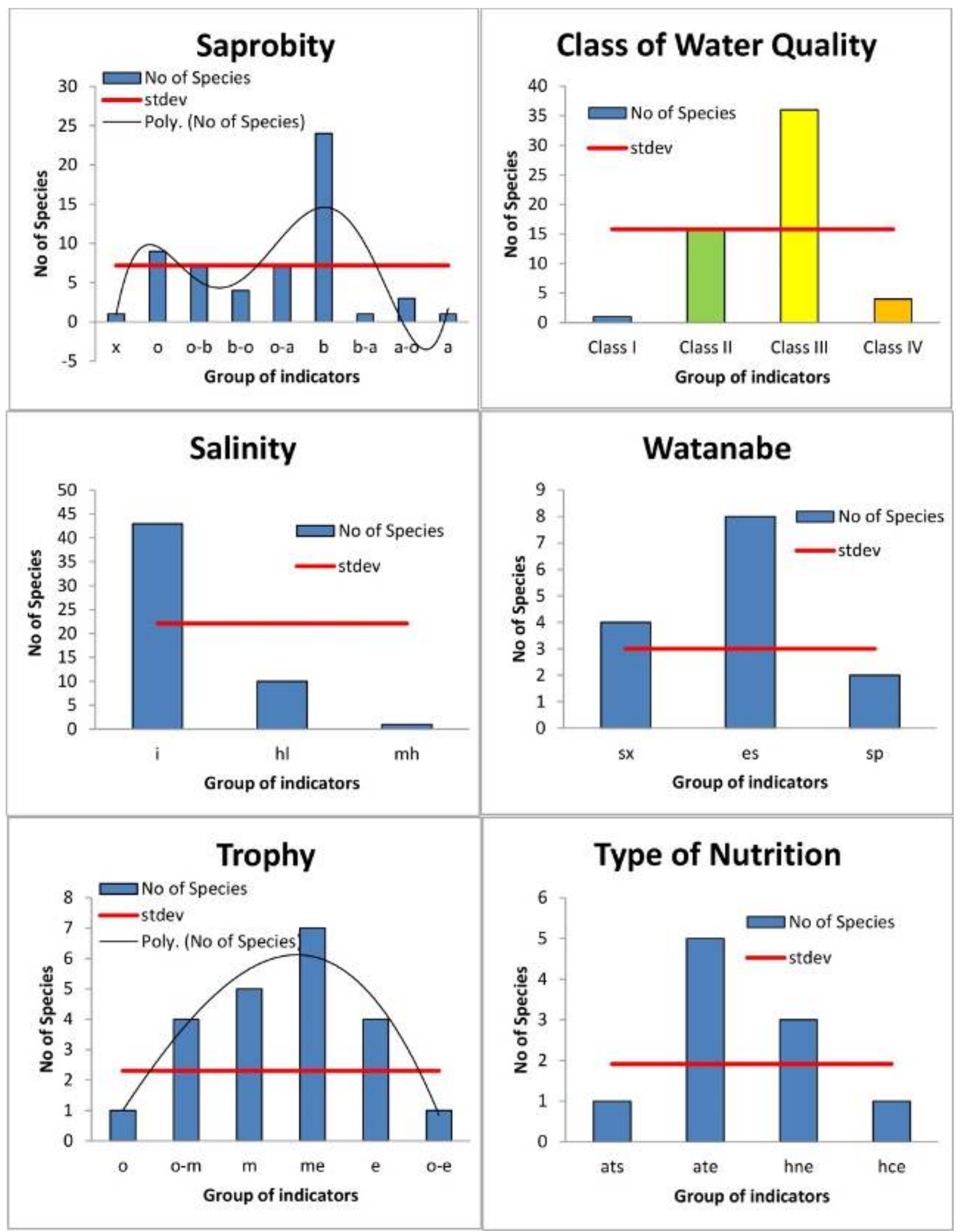

Figure 7. Assessment of the ecological state of the Shardara Reservoir according to phytoplankton species in relation to salinity, organic pollution, trophic status and nutrition type, summer 2015. Water quality classes correspond to the EU color scale. Coloring of the histogram in the figure of water quality classes corresponds to the codes of the EU scale: class I - blue; class II - green; class III - yellow; class IV - orange; class V - red. Abbreviations for indicator groups of algae appear in Table 3. 

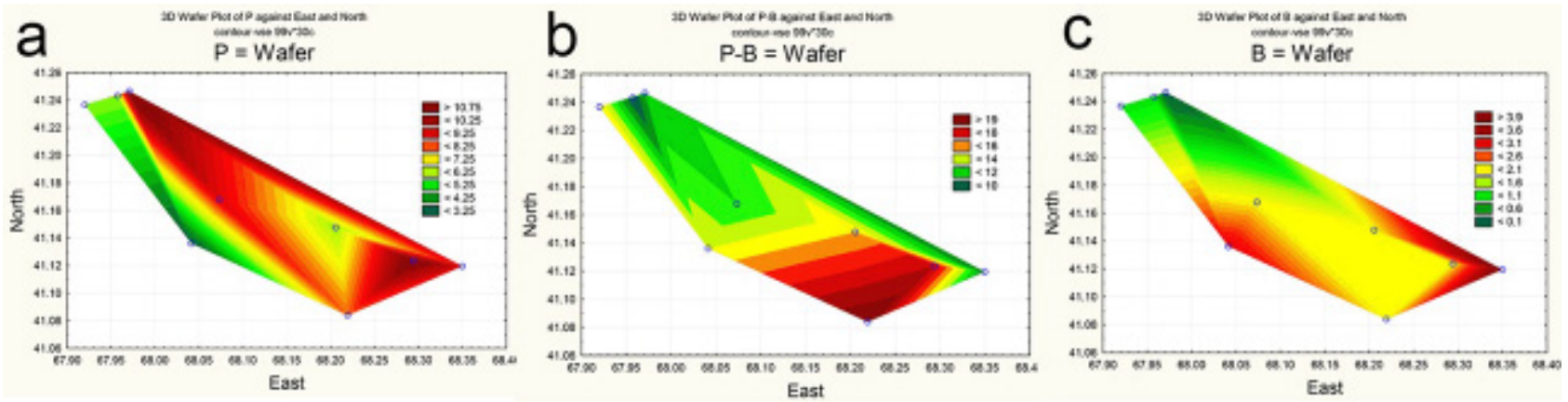

Figure 8. Distribution of algal species of habitat indicators over the water surface of the Shardara Reservoir, summer 2015. Abbreviation of ecological groups of algae as in Table 3: Substrate preferences: $\mathrm{P}$ - planktonic, P-B - plankton-benthic, B - benthic.
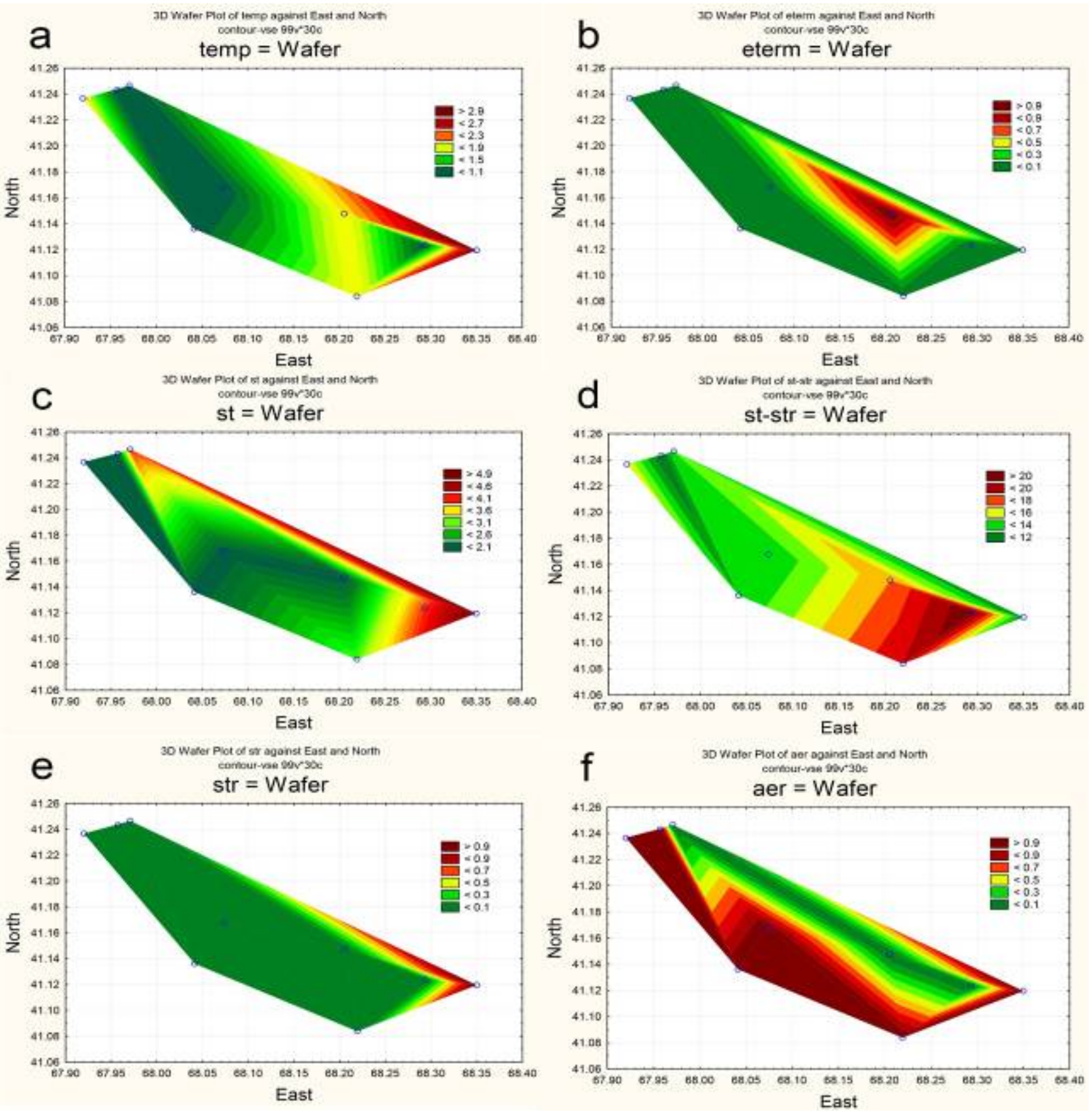

Figure 9. Distribution of the temperature and water oxygenation indicators across the water surface in the Shardara Reservoir, summer 2015. Abbreviation of ecological groups of algae as in Table 3: Temperature preferences: temp - temperate temperature, eterm - eurythermic. Oxygenation and water moving: st - standing water, str - streaming water, st-str - low streaming water, aer - aerophiles. 

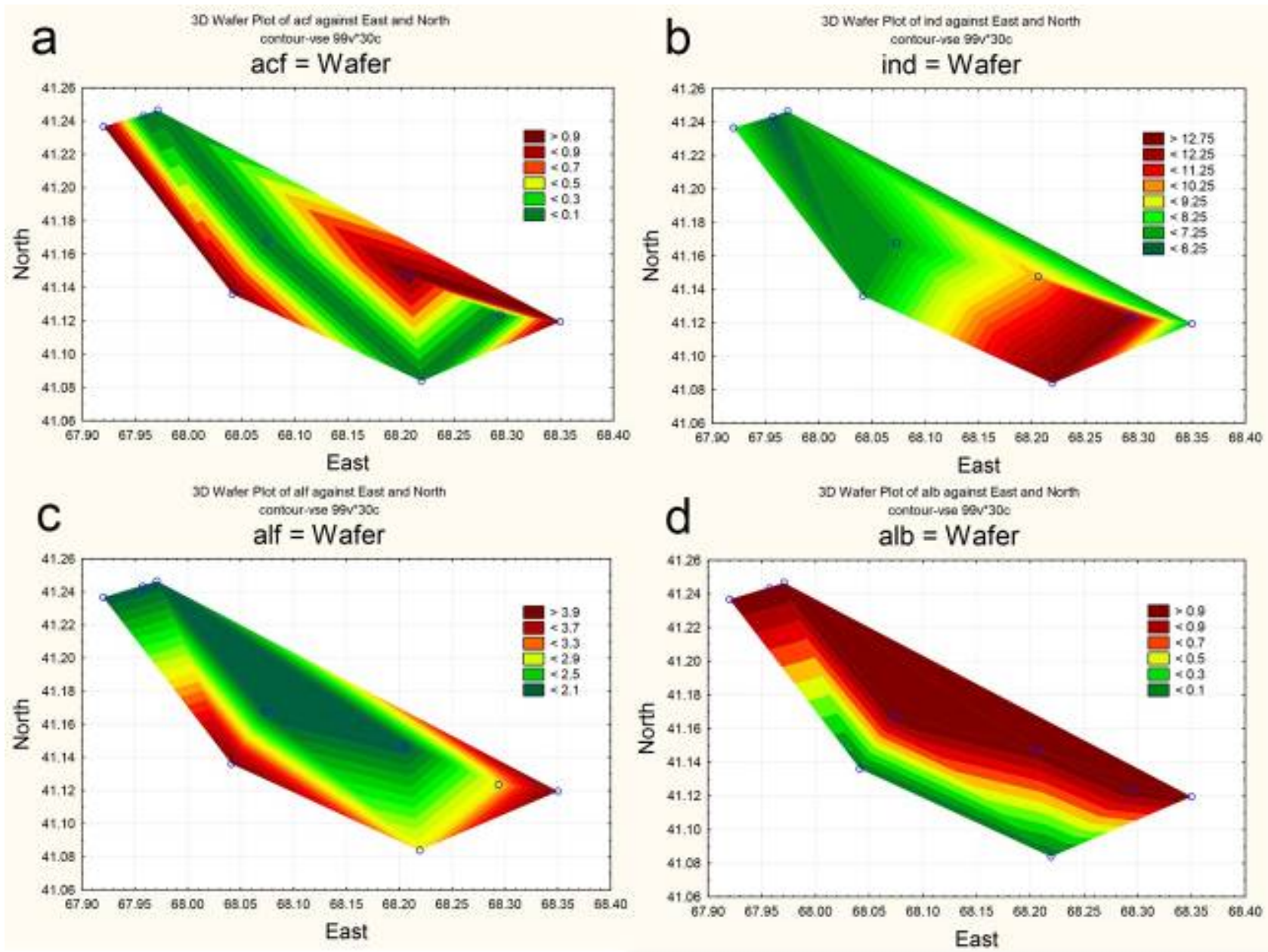

Figure 10. The distribution of algal species indicators of $\mathrm{pH}$ in the water surface of the Shardara Reservoir, summer 2015. Abbreviation of ecological groups of algae as in Table 3: Acidity: alf - alkaliphiles, ind - indifferents, acf - acidophiles, alb - alkalibiontes
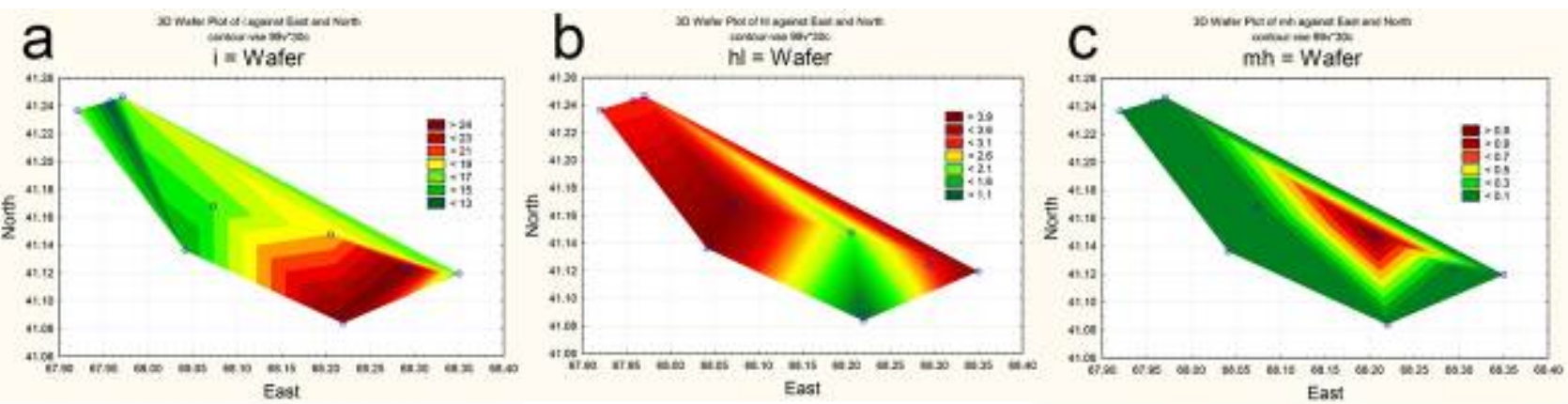

Figure 11. The distribution of algae species - salinity indicators in the water surface of the Shardara Reservoir, summer 2015. Abbreviation of ecological groups of algae as in Table 3: Halobity: i - oligohalobes-indifferent, hl - halophiles, mh - mesohalobes 

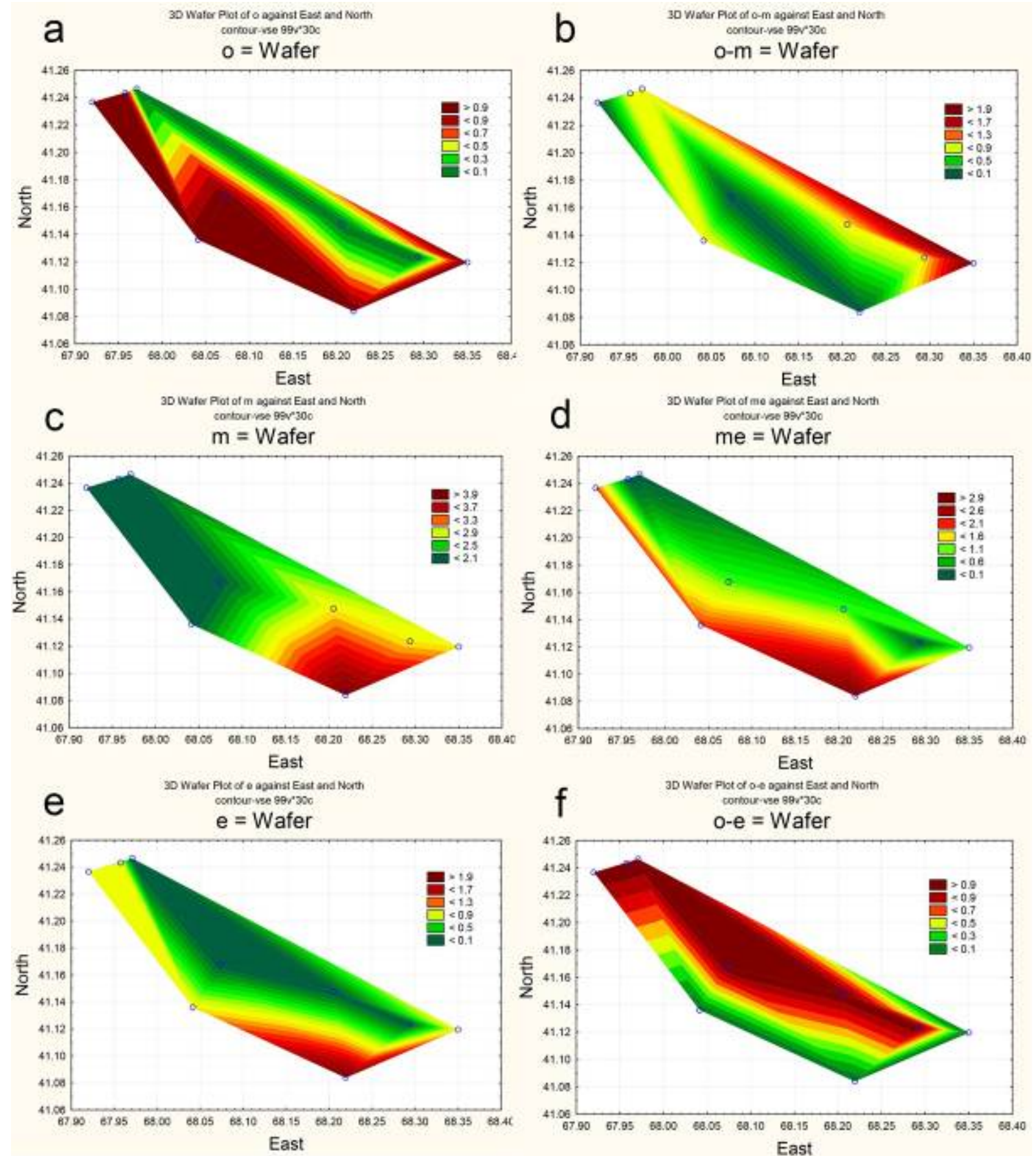

Figure 12. The distribution of algae species - trophic indicators across the water surface in the Shardara Reservoir, summer 2015. Abbreviations of ecological groups of algae as in Table 3: Trophic state: o - oligotraphentic; o-m - oligo-mesotraphentic; $\mathrm{m}$ - mesotraphentic; me - meso-eutraphentic; e eutraphentic; o-e - oligo-eutraphentic. 

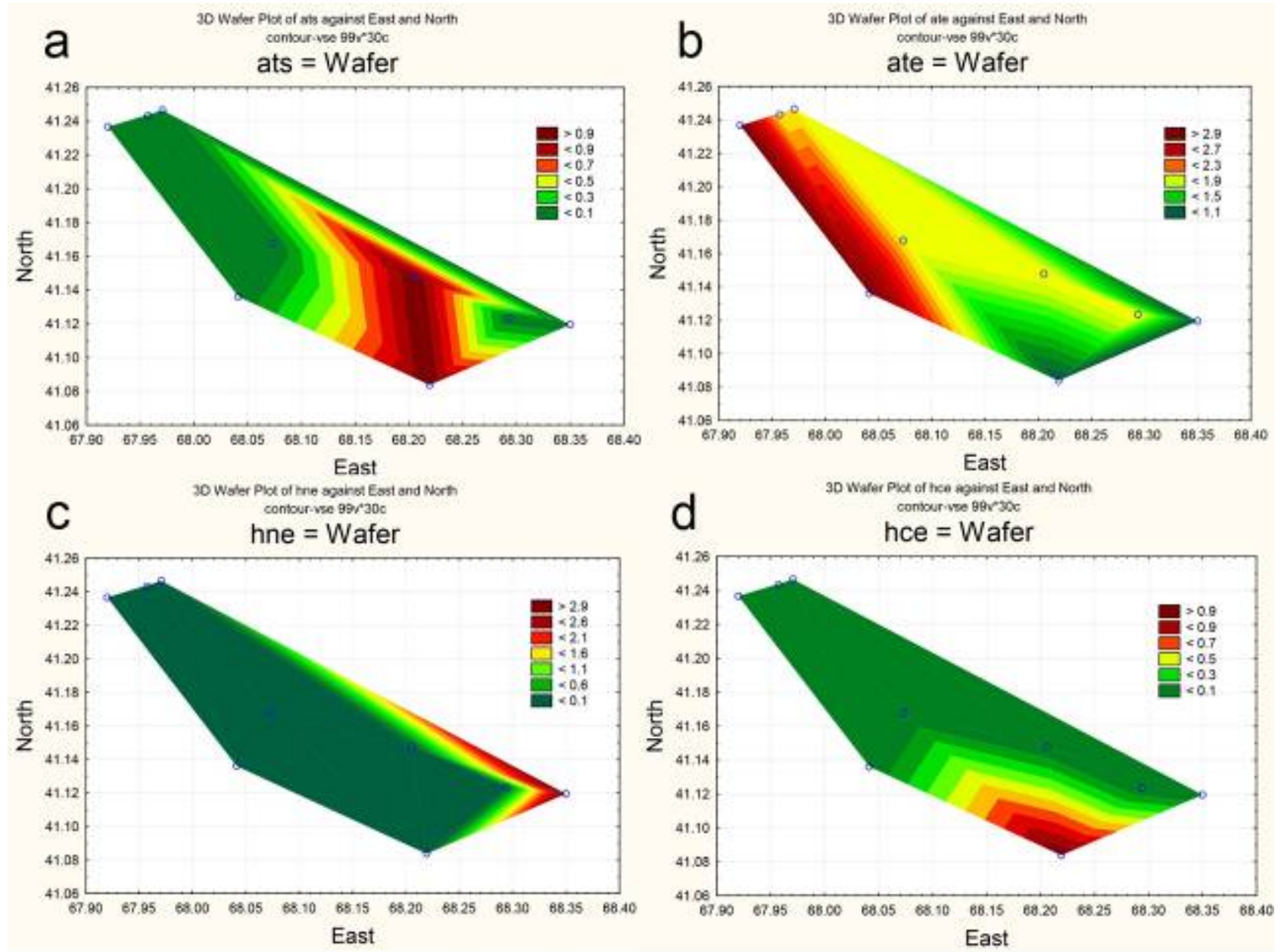

Figure 13. The distribution of algal species - nutrition type indicators across the water surface of the Shardara Reservoir, summer 2015. Legend for ecological groups of algae as in Table 3: Nitrogen uptake metabolism: ats - nitrogen-autotrophic taxa, tolerating very small concentrations of organically bound nitrogen; ate - nitrogen-autotrophic taxa, tolerating elevated concentrations of organically bound nitrogen; hne - facultatively nitrogen-heterotrophic taxa, needing periodically elevated concentrations of organically bound nitrogen; hce - nitrogen-heterotrophic taxa, needing elevated concentrations of organically bound nitrogen. 

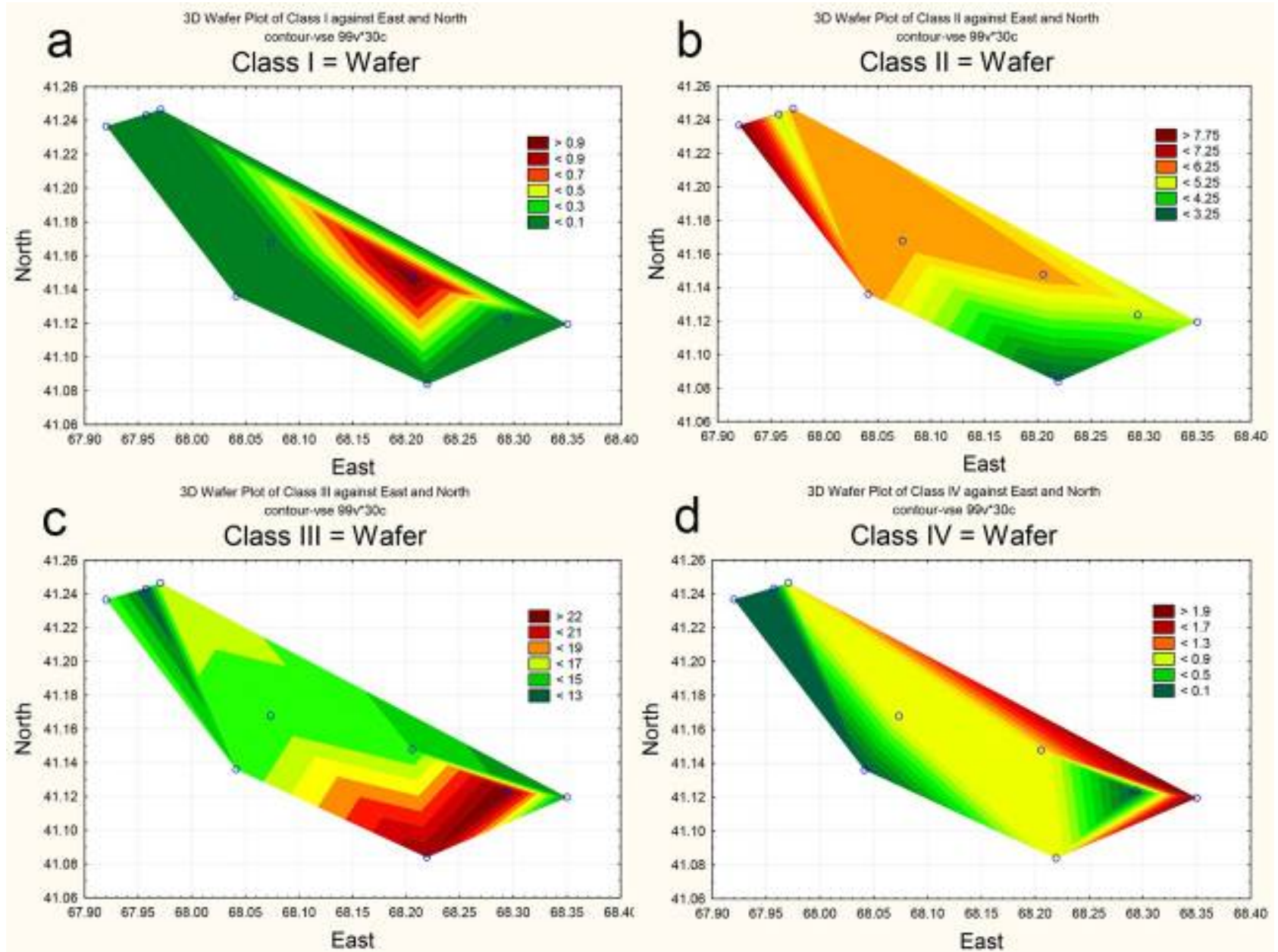

Figure 14. Spatial maps of algal species indicators of water quality class distribution in the Shardara Reservoir, summer 2015

Dendrite of similarity of species composition, drawn up using the Ward method based on Euclidean distance, emphasized the only community (St. 3). It includes the greatest number of species from other communities and represents the floral core of the entire water body (Fig. 5). Therefore, floristically, phytoplankton of the Shardara Reservoir represents the continuum in relation to the number of species in its different parts.

\subsection{Bioindication}

The taxonomic composition of phytoplankton in the Shardara Reservoir was dominated by green algae (Fig. 6). In relation to habitat preferences, benthic forms represented the majority of phytoplankton, and planktonic species were about half that number. In relation to $\mathrm{pH}$ value and amount of dissolved oxygen, phytoplankton was mainly represented by the indifferent-group: species that preferred alkaline waters with $\mathrm{pH}$ values of 9.0-11.0 and tolerated oxygen deficiency came in second.

According to the level of organic pollution, beta-mesosaprobionts dominated in the composition of algal communities. They prefer moderately polluted conditions, Class 3 of water quality (Fig. 7). For the most part, the taxonomic lists of phytoplankton were mainly oligohalobes - indifferent with the small amount of oligohalobes halophiles. Among the diatoms (according Watanabe [25]), most of the species belong to eurysaprobes, with fewer amounts of saproxenes and saprophiles (Fig. 7).

In relation to the trophic level of the reservoir, the distribution of algal species was close to normal with a predominance of mesotraphentic and meso-eutraphentic species (Fig. 7).

Characteristics of algae according to nutrition type showed that the majority of species in the community belonged to autotrophs that were able to withstand very low-organic pollution. In second place were facultative heterotrophs, surviving at low nutrient loads. Approximately equal in number were the obligate (strict) autotrophs and facultative heterotrophs, preferring, respectively, clean and dirty conditions (Fig. 7).

\subsection{Spatial Analysis with the Wafer Surface Plots}

Distribution of the main indicator groups of phytoplankton on the surface of the reservoir was analyzed by means of Statistica 12.0. Figure 8 shows the aligned distribution of planktonic species over the reservoir surface. 
Plankton-benthic inhabitants were concentrated in the southeastern shallow part of the reservoir where macrophytes developed. Benthic species occupied shallow places in the upper part of the reservoir.

Only two groups of water temperature indicators were found in the reservoir community in the very short list of the temperature regime indicators in freshwaters. Figure 9 demonstrated concentration of temperate temperature indicators in the upper part of the reservoir where the rivers flow in (Fig. 9a). The eurythermic species that survived in wide ranges of water temperatures were concentrated in the central part of the reservoir (Fig. 9b). This may be due to the stability of warmer waters during the year in the central part of the reservoir with cooler water input from the rivers Syr-Darya and Keles.

Admittedly, water oxygenation is the parameter that regulates the high levels of trophic pyramid life. Six groups (Fig. 9c-f) represented indicators of water oxygenation in the Shardara Reservoir. Their distribution shows that standing low-oxygenated waters were in the eastern and northern parts of the reservoir (Fig. 9c). Middle oxygenation was found in the whole upper part (Fig. 9d). Indicators of streaming waters with sufficient / ample oxygenation were indicated in the eastern part, within the area of the Syr-Darya River's inflow (Fig. 9e). The group of aerophiles that prefer oxygenated waters was revealed across the reservoir's coastal zone (Fig. 9f). It roughly divided the reservoir's surface into two different parts - well-oxygenated periphery and low-oxygenated northern and central part.

Spatial distribution of the $\mathrm{pH}$ indicators (Fig. 10) showed that low-pH waters are located in the coastal zone with macrophytes (Fig. 10a). The pH-indifferents were mostly in the upper part (Fig. 10b), whereas indicators of alkaline water, such as alkaliphiles and especially alkalibiontes, were concentrated in the coastal zone of the reservoir. This finding demonstrates the influence of the non-point source of alkaline waters coming to the reservoir from the surrounding territory.

It is crucial to identify salinity indicators for the arid zone reservoir, such as the Shardara. Altogether, three groups of salinity indicators were found in the planktonic communities of the reservoir (Fig. 11). It is evident that salinity-indifferent species were distributed mostly in the upper part of the studied reservoir (Fig. 11a). Halophilic species were well distributed across the reservoir surface, excluding the part in the south where agricultural water channels discharge (Fig. 11b). However, the mesohalobes (Fig. 11c) that prefer saline waters were concentrated in the central part of the reservoir, which means that the present level of water salinity can be assessed as the regional norm.

One of the final parts of the assessment is to study the trophic level of the reservoir. Indicators of the reservoir's tropic state represented all six trophic groups (Fig. 12). Oligotropic and oligo-mesotrophic spacies were found in communities across the reservoir coast (Fig. 15a,b). Mesotrophic, meso-eutrophic, and eutrophic species were concentrated in the southern part of reservoir (Figs. 12c-e), whereas the high-trophic level indicators (Fig. 12f) were presented in the central part of the reservoir surface. This picture shows us the relationship of the trophic level with the three parameters - depth of reservoir, its water temperature, and input from the rivers and channels enriched by nutrients.

The final question that we pose in bioindication assessment is - which groups of organisms of the first trophic level utilize the nutrients in reservoir. The response to this question can reveal the mechanism of protein creation as well as the toxic influence on the photosynthetic process. Figure 13 shows the distribution of indicators of nitrogen uptake metabolism. Typical autotrophs were concentrated in the area of the agricultural channels' input in the southern part of reservoir (Fig. 13a). Well-developed autotrophs were presented in the lower part, the deepest part of reservoir (Fig. 13b). Facultative heterotrophs that use dissolved organic matter are concentrated in the area of the inflow from rivers and channels in the upper part of the reservoir (Figs. 13c, d). Thus, this led us to the conclusion that water with some toxicants that impacted the algal photosynthesis comes from the rivers' and agricultural channels' inflow, with the reservoir ecosystem being capable of utilizing and dissolving the toxicant impact at the lower part near the dam.

Indicators of the water quality classes were accumulated from the data of saprobity in Table 3. Each of the saprobity indicators' group has its own saprobity value that relates to one of five classes of water quality. Figure 14 shows that Class I indicators were found in the central part of reservoir (Fig. 14a). Class II indicators were mostly distributed in the lower part of reservoir (Fig. 14b). Class III waters were indicated in the southern part near the agricultural channels' input (Fig. 14c). Moreover, the most polluted waters were coming from the rivers Syr-Darya and Keles, as can be seen in Figure 14d. The graphical model of the reservoir water quality dynamics led us to conclude that the most polluted water comes from the upper watershed, Syr-Darya River and Keles River.

\section{Discussion}

The reservoir is large, about $80 \mathrm{~km}$ in length and $25 \mathrm{~km}$ in width, and represents a major water resource and fishery in the southern arid part of Kazakhstan. It is a transboundary water body with Uzbekistan, which is filled with water from the Syr-Darya River and other rivers with cross-boundary watersheds. Therefore, is very important, not only to monitor its water quality but also to try to find the new cost-effective methods for its assessment. In the Shardara Reservoir ecosystem, we implemented not only an integral bioindication method for assessment but also a new statistical method of surface mapping.

Ecological conditions of the large regional water bodies are numerous. Whereas the Balkhash Lake, even in its western freshened part, is brackish [27], the Kolsay lakes are fresh [28]. The northern Kazakhstan lakes have many ranges of salinity from 0.9 to $30 \mathrm{~g} \mathrm{l}^{-1}$ [29]. As can be seen in 
our recent study, the water salinity of lakes and reservoirs in the south-boreal floristic province varied from $0.5 \mathrm{~g}^{-1}$ in Great Lota in Turkey [30] to $2.2 \mathrm{~g} \mathrm{l}^{-1}$ in the Sasyk Reservoir in Ukraine [31]. The southern subtropical province of India has also freshwater lake Santragachi [32] with $0.3 \mathrm{~g} \mathrm{l}^{-1}$ and the Shibpukur Lake with $4 \mathrm{~g} \mathrm{l}^{-1}$ [33]. In Israel, in the arid zone, the waterbodies' salinity varied from 0.9 to $30 \mathrm{~g} \mathrm{l}^{-1}$, like in northern Kazakhstan [29]. The data led us to conclude that the Shardara Reservoir with $1.1 \mathrm{~g} \mathrm{l}^{-1}$ is within the regional norm in its freshwater range.

The studied reservoir's ecosystem's algal species richness of phytoplankton was represented by 76 species and is in the same framework as other regional waterbodies where the number of species varied from 4 (Maybalyk) to 64 (Kojbagor) [34]. It is remarkable that species richness in the abovementioned lakes decreased with increased salinity, which is characteristic of lakes with wide ranges of salinity $[29,35]$. Therefore, the Shardara's diversity confirms its fresh water is sustainable on the scale of regional lakes.

The domination of the Chlorophyta species among the Shardara phytoplankton characterizes its ecosystem as effective, like the large shallow Sasyk Reservoir [31], or subtropical lakes in India [32, 33]. This result is beyond the scope of regional characteristics of water bodies, where diatoms are the dominants in phytoplankton [27-30,34].

Bioindication methods were implemented for the first time for the Shardara Reservoir water quality analysis, as it was done for the abovementioned regional lakes [28, 29, 34]. Plankto-benthic inhabitants represent the majority of algal species that were found in the Shardara Reservoir's phytoplankton. Indicators revealed alkaline, medium-oxygenated, and medium organically polluted water of Class III water quality. The reservoir water can be characterized as mesotrophic by algal indicators of trophic state, mostly diatoms. Algae that inhabited the whole reservoir's water mass preferred the photosynthetic process of protein synthesis in the nutrient enriched waters.

In comparison with the other regional lakes' bioindication results, the Shardara Reservoir environment showed a similar tendency in the water quality forming process with salinity as the regulating factor, like in Northern Kazakhstan and Kolsay lakes [28,34], as well as in Ukraine [31], Turkey [30], and Israel [29]. The bioindication assessment results were different from the Indian subtropical lakes in that the water temperature is the major regulating factor $[32,33]$.

New statistical approaches to the surface mapping of the bioindication results revealed important variables for monitoring the Shardara ecosystem and in finding the sources of water pollution. In the first step of the map construction variables or indicator groups should be selected to use for the mapping. In recent work [31], we chose the factors intuitively. Now, with the help of the Statistica 12.0 program, we constructed a set of surface maps for each indicator group as well as for species richness in taxonomic divisions.

As a result, by using spatial mapping, the most important groups of bioindicators as well as the parts of the reservoir that point to the pollution source were revealed.

Therefore, even though the reservoir water in the summer period had the highest temperature in the eastern shallow part, the eurythermic species that survive in a wide range of water temperatures were concentrated in the central part of the reservoir. The data indicated the long-term maintenance of high-temperature water in the center of the reservoir water mass during the year. A similar situation was recorded in the sustainability assessment of subtropical lakes with respect to water temperature, such as with the Santragachi or Shibpukur lakes $[33,36]$. Remarkably, in the studied reservoir as well as in these lakes, the Chlorophyta algae dominated. The green algae have the best conditions to flourish in warm water, indicating that temperature is one of the major regulating variables of the Shardara ecosystem.

The surface mapping of the reservoir's water oxygenation indicators shows differences between the central part and coastal zone. Maps roughly divide the reservoir surface into two parts - well oxygenated periphery and low-oxygenated northern and central parts. In the Ukrainian Sasyk Reservoir [31] it can be seen that the coastal zone was also well-oxygenated whereas we cannot be compared our correlation of well oxygenated water and coastal zone of other regional lakes and reservoirs because surface indication was not implemented there. We assumed that in both cases, in the Sasyk and Shardara reservoirs, the differences in water oxygenation indicators could be related to the wind influence on the large water surface when the wind creates waves in the coastal zone and enriches the water with oxygen.

Maps of water $\mathrm{pH}$ indicators show the influence of the non-point source of alkaline waters coming to the reservoir from the surrounding territory. A similar situation was revealed in the Sasyk Reservoir [31], also with the help of $\mathrm{pH}$ indicators only.

There were no differences in the map of water salinity indication from the regional norm, like it was in other water bodies in Kazakhstan [28, 34], as well as in Ukraine [31], Turkey [30], and Israel [29].

The indication maps of the trophic level of the Shardara Reservoir show the relation of the trophic level to three parameters - the depth of the reservoir (if low - the trophy increased), the water temperature (if high - the trophy increased) and the inflowing waters of the rivers and channels enriched by nutrients (in the coastal zone). A similar situation was revealed in the Sasyk Reservoir where the coastal zone and the river mouth were indicated as the source of pollution [31]. The eutrophication source analysis is closely related to the water quality indication. Maps of the water quality indication in the reservoir show that the most polluted waters come from the upper watershed with the water of Syr-Darya and Keles rivers. A similar situation was revealed in the Sasyk water reservoir of southern Ukraine [31] where pollution comes with the Sarata and Kogylnik rivers (it should be noted that the indication was made in the same summer period, and the river had only 
underground flow).

One of the crucial questions was, "how do the first trophic level organisms utilize the nutrients in the reservoir"? Surface maps illustrate that nitrogen-uptake metabolism indicators are distributed across the reservoir unevenly. The prevalence of heterotrophic species points out to some toxic influence on the reservoir ecosystem. Surface maps of the distribution of nutrition type groups identified the inflowing water of rivers and agricultural channels as the main source of toxic substances that stress the photosynthetic algae. The same was true for the Sasyk Reservoir [31]. At the same time, the maps demonstrated a high level of self-purification capability of the reservoir ecosystem as well as the utilization of the toxicants in the area near the dam in contrast with the Ukrainian reservoir. The surface distribution analysis of diverse biological, bioindicational and environmental variables with help of statistically generated maps is very easy and demonstrated most effective way than early used bulky mapping approaches [5-8,31].

\section{Conclusions}

In this research, we used the new statistical approach to analyze the data on biodiversity and phytoplankton productivity for the first time. The Statistica 12.0 program was used for construction of spatial maps for different variables together with bioindication methods. Improvement in the spatial analysis of the ecosystem's relationship to environmental variables can only be done with the use of ecological maps.

One of the major features of our maps is the continuous parameter distribution that can be used for future analysis and prognostic purposes. In any case, the previously constructed maps for the Ukrainian Sasyk Reservoir are similar to the study done here on phytoplankton abundance and biomass. The same applies to the analysis of water salinity, environment, and indication assessment, all of which are relevant for future studies of the Shardara Reservoir as the major water source in the arid region of southern Kazakhstan.

It is not necessary to choose specific data for this approach in mapping now. It gives us the full picture of all data on spatial analysis. In our analysis, a very small range of data can be used to point out the gradients between parts of the studied reservoir. As an introduction to the new surface mapping method, we conclude that statistical maps are more representative and correspond well to the environment.

The maps helped us to characterize the Shardara Reservoir as fresh water, with the source of pollution coming from the rivers and agricultural channels together with some toxic substances that are dissolved at the lower part of the waterbody near the dam. The main self-purification processes in the studied waterbody mostly occurred by means of the green planktonic algae at the central part of the reservoir.

The present study demonstrated that the use of surface mapping method is advisable when assessing the ecological conditions of the water body not through individual stations but for the whole reservoir without dividing it into stations. This study confirmed our bioindication results on the correlation of water temperature and total dissolved solids, which is strongly related to water mass disturbance by the wind. Statistical mapping also confirmed that the bioindication method showed a more detailed picture than the standard physico-chemical analysis.

Thus, the bioindication and chemical data surface mapping represent practical and easy-to-use tools for data comparison that can be included in the monitoring of the Shardara Reservoir.

\section{Acknowledgements}

The work was carried out under the project № 1846/ГФ4 Г.2015-Г2016 for Committee of Science, Ministry of Education and Science, Republic of Kazakhstan "Development of the methods for controlling the ecological state of water bodies in Kazakhstan" as well as partly supported by the Israeli Ministry of Absorption.

\section{REFERENCES}

[1] T. Y. Lopareva, N. A. Amirgaliev. Ionic and salt composition and some technical properties of the water in Shardara reservoir. In: Fish resources of water bodies of Kazakhstan and their use, Kainar, Almaty, 1973.

[2] A. S. Malinovskaya, B.A. Ten. Hydrofauna of the reservoirs in Kazakhstan, NaukaKazSSR, Almaty, 1983.

[3] N. A. Amirgaliev, Zh. B. Ismailova, F. E. Tagaeva, S. T. Nakupbekov. Hydrochemical indicators and the level of pesticide contamination of Shardara reservoir ecosystem. In: Ecosystems and fish resources of reservoirs Kazakhstan, Bastau, Almaty, 1995.

[4] N. A. Amirgaliev. Aral and Syr Darya basin: hydrochemistry, water toxicology issues, Bastau, Almaty, 2007.

[5] S. Barinova. Algal diversity dynamics, ecological assessment, and monitoring in the river ecosystems of the eastern Mediterranean, Nova Science Publishers, New York, USA, 2011.

[6] International Fund for Agricultural Development. Good practices in participatory mapping: A review prepared for the International Fund for Agricultural Development, Online available from http://www.ifad.org/pub/map/PM_web.pdf

[7] D. Corbett, G. Rambaldi, P. A. K. Kyem, D. Weiner, R. Olson, J. Muchemi, M. McCall, R. Chambers. Overview: mapping for change - the emergence of a new practice, Online available from http://www.iapad.org/publications/ppg is/ch01_overview_pp13-19.pdf

[8] G. Rambaldi, P. A. K. Kyem, M. McCall, D. Weiner. 
Participatory spatial information management and communication in developing countries, Online available from http://www.ejisdc.org/ojs2/index.php/ejisdc/article/vie wFile $/ 237 / 158$

[9] Guideline for chemical analysis of surface water, Gidrometeoizdat, Leningrad, 1977. (In Russian).

[10] G. S. Fomin. Water. Control of chemical, bacterial and radiation safety according to international standards, NGO "Alternative", Moscow, 1995. (In Russian)

[11] I. A. Kisselev. Methods of study of plankton. In: Life of freshwaters of the USSR, USSR Academy of Sciences, Moscow, Leningrad, 1956. (In Russian).

[12] M. M. Gollerbach, E. K. Kossinskaya, V. I. Polyansky. Key to freshwater algae USSR, Vol. 2, Blue-green algae, Soviet Science, Moscow, 1953. (In Russian).

[13] M. M. Zabelina, I. A. Kisselev, A. I. Proshkina-Lavrenko, V. S. Sheshukova. Key to freshwater algae of the USSR, Vol. 4, Diatoms, Soviet Science, Moscow, 1951. (In Russian).

[14] T. G. Popova. Key to freshwater algae of USSR, Vol. 7, Euglenophyta, Soviet Science, Moscow, 1955. (in Russian).

[15] N. A. Moshkova, M. M. Gollerbach. Key to freshwater algae of the USSR, Vol. 10(1), Green algae, Class Ulotriksovye, Ulotriksovye. Soviet Science, Moscow, 1986. (In Russian).

[16] G. M. Palamar-Mordvintseva. Key to freshwater algae of the USSR, Vol. 1(2), Green algae, Class conjugates, Desmidievye (2), Soviet Science, Moscow, 1982. (In Russian).

[17] A. M. Matvienko. Key to freshwater algae of the USSR, Vol. 3, Golden algae, Soviet Science, Moscow, 1954. (In Russian).

[18] S. S. Barinova, L. A. Medvedeva, O. V. Anissimova. Diversity of algal indicators in environmental assessment, Pilies Studio, Tel Aviv, 2006.

[19] V. Heywood. Modern approaches to floristics and their impact on the region of SW Asia, Turk. J. Bot, Vol. 28, 7-16, 2004.

[20] A. B. Novakovsky. Abilities and base principles of program module "GRAPHS", Scientific Reports of Komi Scientific Center, Ural Division of the Russian Academy of Sciences, Vol. 27, 1-28, 2004.

[21] T. V. Guseva (Ed.). Hydrochemical indicators of the environment, Socio-ecological Union, Moscow, 2002. (in Russian).

[22] G. P. Bespamyatnov, Y. A. Krotov. Maximum permissible concentrations of chemicals in the environment, Chemistry, Leningrad, 1985. (In Russian).

[23] F. Hustedt. Die Diatomeenflora des Flußsystems der Weser imGebiet der Hansestadt Bremen, Abhandl. NaturwisVer, Bremen, 34, 181-440, 1957.

[24] F. Hustedt. Systematische und ÖkologischeUntersuchungen über die Diatomeenflora von Java, Bali und Sumatra, Arch. Hydrobiol, Suppl. 15, 131-177, 393-506, 638-790; 16, 1-155, 274-394, 1938-1939.

[25] T. Watanabe, K. Asai, A. Houki. Numerical estimation to organic pollution of flowing water by using the epilithic diatom assemblage - Diatom Assemblage Index (DAIpo), Science of Total Environment, Vol. 55, 209-218, 1986.

[26] H. Van Dam, A. Martens, J. Sinkeldam. A coded checklist and ecological indicator values of freshwater diatoms from the Netherlands, Netherlands J. Aquatic. Ecol., Vol. 28, No. 1, 117-133, 1994.

[27] E. Krupa, G. Slyvinskiy, S. Barinova. The Effect of Climatic Factors on the Long-term Dynamics of Aquatic Ecosystem of the Balkhash Lake (Kazakhstan, Central Asia), Advanced Studies in Biology, Vol. 6, No.3, 115-136, 2014.

[28] E. G. Krupa, S. S. Barinova. Environmental variables regulating the phytoplankton structure in high mountain lakes, Research Journal of Pharmaceutical, Biological and Chemical Sciences, Vol. 7, No. 4, 1251-1261, 2016.

[29] S. S. Barinova, T. M. Bragina, E. Nevo. Algal species diversity of arid region lakes in Kazakhstan and Israel, Community Ecology, Vol. 10. No. 1, 7-16, 2009.

[30] S. Barinova, R. Sivaci. Experimental approach to a lake ecosystem assessment in the Great Lota, Turkey, The Experiment, Vol. 9, No. 4, 566-586, 2013.

[31] O. P. Bilous, S. S. Barinova, N. O. Ivanova, O. A. Huliaieva. The use of phytoplankton as an indicator of internal hydrodynamics of a large seaside reservoir-case of the Sasyk Reservoir, Ukraine, Ecohydrol. Hydrobiol., Vol. 16, 160-174, 2016.

[32] S. Ghosh, S. Barinova, J. P. Keshri. Diversity and seasonal variation of phytoplankton community in the Santragachi Lake, West Bengal, India, QScinece Connect, Vol. 3, 1-19, 2012 .

[33] S. Barinova, R. Sivaci. Experimental approach to a lake ecosystem assessment in the Great Lota, Turkey, The Experiment, Vol. 9, No. 4, 566-586, 2013.

[34] S. S. Barinova, E. Nevo, T. M. Bragina. Ecological assessment of wetland ecosystems of northern Kazakhstan on the basis of hydrochemistry and algal biodiversity, Acta Botanica Croatica, Vol. 70, No. 2, 215-244, 2011.

[35] V. Klymiuk, S. Barinova, A. Fatiukha. Algal bio-indication in assessment of hydrological impact on ecosystem in wetlands of "Slavyansky Resort", Transylv. Rev. Syst. Ecol. Res, the Wetlands Diversity, Vol. 17, No. 1, 63-70, 2015.

[36] S. Barinova, J.P. Keshri, S. Ghosh, J. Sikdar. The influence of the monsoon climate on phytoplankton in the Shibpukur pool of Shiva temple in Burdwan, West Bengal, India, Limnological Review, Vol. 2, No. 2, 47-63, 2012. 